\title{
1 Astrocytes encode complex behaviorally relevant information
}

2

3

4 Katharina Merten ${ }^{1, *}$, Robert W. Folk $^{1}$, Daniela Duarte ${ }^{1}$ and Axel Nimmerjahn ${ }^{1, *}$

$5 \quad{ }^{1}$ Waitt Advanced Biophotonics Center, The Salk Institute for Biological Studies, La Jolla, CA

6 92037, USA

$7 \quad{ }^{*}$ Correspondence: kmerten@salk.edu (K.M.), animmerj@salk.edu (A.N.)
9

\section{Abstract}

Astrocytes, glial cells of the central nervous system, help to regulate neural circuit operation and adaptation. They exhibit complex forms of chemical excitation, most prominently calcium transients, evoked by neuromodulator and -transmitter receptor activation ${ }^{1-4}$. However, whether and how astrocytes contribute to cortical processing of complex behavior remains unknown ${ }^{1}$. One of the puzzling features of astrocyte calcium transients is the high degree of variability in their spatial and temporal patterns under behaving conditions. Here, we provide mechanistic links between astrocytes' activity patterns, molecular signaling, and behavioral cognitive and motor activity variables by employing a visual detection task that allows for in vivo calcium imaging, robust statistical analyses, and machine learning approaches. We show that trial type and performance levels deterministically shape astrocytes' spatial and temporal response properties. Astrocytes encode the animals' decision, reward, and sensory properties. Our error analysis confirms that astrocytes carry behaviorally relevant information depending on and complementing neuronal coding. We also report that cell-intrinsic mechanisms curb astrocyte calcium activity. Additionally, we show that motor activity-related parameters strongly impact astrocyte responses and must be considered in sensorimotor study designs. Our data inform and constrain current models of astrocytes' contribution to complex behavior and brain computation beyond their established homeostatic and metabolic roles. 


\section{Introduction}

29 Mounting evidence from multiple species and central nervous system regions suggests that astrocytes play pivotal roles in neural circuit function and behavior ${ }^{2,3,5}$. Although not electrically

31 excitable, astrocytes display a complex repertoire of intracellular signaling, most prominently

32 calcium transients, triggered by neurotransmitter and neuromodulator receptor activation on

33 their surface. This signaling spans multiple spatial and temporal scales, from sub-second

34 transients in single astrocytes to seconds- or even minutes-long transients in astrocytic networks, suggesting that astrocytes may carry out computations on various timescales related to sensory processing, brain state modulation, and memory formation ${ }^{1,2,4}$.

Despite the recent technical progress in measuring neuronal, astrocyte, and transmitter dynamics in behaving animals, a key unresolved question is precisely how astrocyte excitation relates to animal behavior and how it may contribute to brain computation of cognitive functions.

41 This knowledge gap is partly due to the lack of standardized quantitative behavioral assays that

42 allow tight control over the animal's behavior and associated cellular and molecular signaling.

43 Additionally, data analysis approaches are often based on manually drawn regions of interest,

44 which are poorly suited to capture the complexity of astrocyte excitation or its relationship to

45 circuit dynamics and behavior ${ }^{6,7}$. Moreover, recent studies reporting astrocytic encoding of

46 spatial information ${ }^{8}$ or reward location $^{9}$ in the hippocampus have neglected the impact of mouse

47 motor behavior on astrocyte responses. Astrocytes are known to exhibit widespread calcium

48 excitation during locomotion mediated by local neurotransmitter and volumetric neuromodulator

49 release ${ }^{10-12}$. Therefore, run parameters, particularly the timing of astrocyte response onset

50 relative to run onset and the period between runs, might strongly influence experimental results 51 and interpretation. 
53 Using a quantitative visual detection task, in vivo calcium imaging, robust statistical analyses

54 that account for the joint influence of run and cognitive parameters, and machine learning

55 approaches, we show that astrocyte population transients ("syncytium responses") in the mouse

56 motor cortex are deterministic and encode information about the stimulus, trial type, reward,

57 decision, and the animal's performance level. Astrocyte responses were also significantly linked

58 to run onset, run duration, and inter-run interval. Additionally, we show that astrocyte population

59 responses underlie intrinsic constraints. Our data provide insight into fundamental computations

60 within astrocyte networks and the integration and transformation of molecular signals within their

61 environment, suggesting that these cells contribute to complex behavior and brain computation

62 beyond their established homeostatic and metabolic roles.

\section{Results}

65 To investigate whether astrocytes contribute to cortical information processing and the encoding of complex behavior, we recorded their activity in a visual detection task. This behavioral assay

67 involved numerous trial repetitions across multiple sessions and allowed robust regression and decoding analysis. In total, we recorded 4,837 trials across 21 behavioral sessions (see Methods). Mice were trained to report the presence or absence of a visual stimulus by running on a spherical treadmill for a fluid reward (Fig. 1a,b). Stimuli were presented at two intensity

71 levels: i) salient and ii) close to the animal's perceptual level. The internal state of the mouse

72 determined whether it had seen the stimulus ('yes' decision if the animal initiated a run during

73 stimulus presentation; 'no' decision if it stood still for $>3 \mathrm{~s}$ ). Decision outcomes were classified

74 according to signal detection theory (Fig. 1c). Before stimulus presentation, the mice were

75 required to stand still for $20 \mathrm{~s}$. If mice interrupted the stand-still phase, the trial was aborted and

76 counted as a spontaneous run. Fig. 1d shows trial outcome proportions for an example session.

77 To create psychometric detection curves for each animal, we used the proportion of 'yes' 
78 decisions for the two stimulus intensities (Fig. 1e). The mice were rewarded on correct trials

79 only (hits and correct rejections). Trials with stimuli close to the perceptual threshold, in which

80 the animals did not detect the stimulus, were not reinforced. This reward contingency led to a

81 slight bias of the mice to erroneously report the presence of a stimulus in some of the stimulus

82 absent trials (Fig. 1e). The animals' performance levels varied within and across sessions. We

83 computed a measure of discriminability (d') derived from signal detection theory ${ }^{13}$ by subtracting

84 z-scores (normal deviates) of median 'hit' rates from z-scores of median 'false alarm' rates

85 (Fig. 1f). Performance intervals exceeding the detection threshold were considered high-

86 performance states, whereas periods with d'<2 were classified as low-performance states.

Astrocyte calcium activity in fully trained GFAP-GCaMP6f mice was recorded using two-photon imaging (Fig. 1g). All recordings were performed in cortical layer $2 / 3$ of the primary and secondary motor areas (M1/M2) and had a $\sim 510 \times 640 \mu \mathrm{m}$ field-of-view recorded at $\sim 30.9 \mathrm{~Hz}$ (Fig. 1g,h). While the GFAP promoter drives expression in most and predominantly astrocytes,

92 a limited region-dependent neuronal expression has been reported ${ }^{14,15}$. We, therefore,

93 computationally identified and excluded any areas showing features of neuronal activity ${ }^{16}$ (see

\section{Methods).}

96 Next, we analyzed the animals' task-related running responses. During hit trials and false

97 alarms (FA), the runs started shortly after stimulus presentation (mean reaction time of two

98 representative mice: $0.7 \mathrm{~s}$ and $1.2 \mathrm{~s}$ ) (Fig. $2 \mathrm{a}$, top panel). During correct rejections (CR) and

99 miss trials, mice remained still on the treadmill during the stimulus phase. However, during

100 reward consumption, $97.6 \%$ of CR trials were followed by a run. Similarly, stimulus offset

101 triggered runs in $7.2 \%$ of the miss trials. 
103 To study astrocytes' encoding of complex cognitive functions in the context of running, we only 104 analyzed trials that included runs with comparable characteristics (for trial selection criteria and 105 numbers, see Methods and Table 1). Moreover, we applied multivariate analysis to explore the 106 joint influence of cognitive and run-related variables and determine the effect of each variable in 107 the presence of the others. To analyze astrocytes' response properties, we implemented a 108 Region of Activity (ROA) analysis algorithm that uses three-dimensional filtering and noise109 based thresholding on individual pixels over time to detect significant fluorescence transients ${ }^{7}$

110 (see Methods). Astrocytic syncytium responses were plotted as the percentage of active pixels 111 within the labeled area over time (Extended Data Fig. 1a). We characterized the syncytium 112 responses by calculating their onset (relative to run onset), duration, peak value and time, and 113 offset (Extended Data Fig. 1b). We also calculated the total extent of activation (i.e., projection 114 of active pixels throughout the response interval normalized to the total labeled area) and mean duration of pixels activated during the response interval. To identify the contribution of behavioral variables (trial type, performance level, recording area, mouse identity, current and

117 previous run parameters) to astrocyte syncytium response characteristics, we used multivariate 118 linear mixed-effects (LME) models with recording sessions as a random effect (see Methods).

120 We found that behavioral context significantly influenced the astrocyte syncytium response to 121 running. Not every run was capable of triggering an astrocyte response. In areas M1/M2, we 122 found a significant effect of inter-run interval period on the probability of eliciting an astrocyte 123 response (Extended Data Fig. 2a), a cell-intrinsic mechanism previously reported for cerebellar 124 Bergmann glia ${ }^{10}$. The shorter the rest period, the less probable (Extended Data Fig. 2b), 125 weaker (Extended Data Fig. 2d), and more delayed astrocyte responses were (Extended Data 126 Fig. 2c). Nevertheless, the trial type had a significant effect on the probability of astrocyte 127 syncytium responses. To further investigate this trial type effect, we focused on task-related 
runs with $\geq 20$ s stand-still phase (by task design) and spontaneous runs with $\geq 15$ s inter-run

distance. Notably, astrocyte syncytium response probability was significantly higher for rewarded runs than spontaneously initiated runs (Fig. 2b).

Next, we aligned all run trial transients at the run onset to examine how syncytium responses depended on behavioral context parameters. This representation also allowed us to compare

134 task-related trials to spontaneous runs (Fig. 2c). Averaged syncytium transients lasted $\sim 10 \mathrm{~s}$, 135 and their onset latency (3 s) was strongly correlated to run onset (Fig. 2d). We found

136 significantly shorter onset latencies (2.7 s) for hit compared to CR trials (3.2 s) and spontaneous

137 runs (3.2 s) (Table 3). Additionally, our LME analysis revealed that astrocyte syncytium signals significantly encoded the detection decision, with earlier onsets for hit and FA trials ('yes' decision) (Fig. 2e, Table 9). Applying the LME model on hit trials only, we found that syncytium responses to salient stimuli were shorter (2.6 s) than to threshold stimuli (3 s) (Fig. 2f,

Table 10). The strength of astrocyte syncytium responses (i.e., its response duration, peak, total extent of activation, and mean pixel activation duration) was similar for rewarded trials (hits and CRs) (Fig. 2g) but stronger compared to spontaneous runs. Astrocyte syncytium responses

144 also significantly differed between rewarded and error trials, with correct trials showing longer

145 response durations, larger total extent, and longer mean pixel activation duration (Fig. 2h).

146 Astrocyte calcium activity also significantly encoded the animals' performance levels throughout

147 the session. The response peaks, total extent, and the mean pixel activation duration were 148 significantly larger/longer during low-performance periods than high-performance phases

149 (Fig. 2i). Finally, responses in area M1 showed more prominent peaks, total extent, and mean

150 pixel activation duration than in area M2 (Extended Data Fig. 3). In summary, astrocyte

151 syncytium responses are extraordinarily versatile, with different response characteristics 152 encoding various behavioral features. 
154 Notably, our LME model also revealed a significant dependence of the astrocyte syncytium

155 response duration on the current run duration with longer runs resulting in slightly longer

156 response durations $(19 \%, 12 \%$, and $35 \%$ slope for hits, CRs, and spontaneous runs,

157 respectively; Extended Data Fig. 4a, Table 12). To examine this dependency more closely, we

158 plotted astrocyte syncytium responses for different run durations (1-10 s, 10-15 s, 15-20 s, 20-

$15930 \mathrm{~s}$ ) for the hit and CR trials and spontaneous runs (Fig. 3a-c). For rewarded trials, run

160 duration did not affect response onset. In contrast, longer run durations for spontaneous runs

161 resulted in longer response latencies (42\% slope, Fig. 3d, Table 13). Likewise, while the

162 response peak location shifted only slightly toward later time points for longer runs in hit (4\%

163 slope) and CR trials (11\% slope), we found a considerable peak location shift (66\% slope) for

164 spontaneous runs (Fig. 3e, Table 14). Additionally, when we calculated response offsets

165 relative to the run onset, this duration increased only slightly with run duration for rewarded trials

166 (18\% increase for hit trials and $16 \%$ for CR trials). In comparison, it changed drastically for

167 spontaneous runs (75\% slope) (Fig. 3f, Table 15). These findings imply that different

168 mechanisms control the on- and offset of astrocyte syncytium responses in different behavioral

169 contexts.

171 Rewarded runs appeared to have a defined onset and offset period of $\sim 15 \mathrm{~s}$, within which the

172 response peak and duration varied slightly. We calculated the difference between the response

173 offset and run offset to investigate whether a behavioral event might trigger the astrocytic

174 syncytium response offset. For rewarded trials, the response offset coincided with run offset for

175 13-15 s-long runs (Extended Data Fig. 4b, top and center panels). For shorter runs ( $<13 \mathrm{~s})$, the

176 response duration outlasted the run, and for longer runs (>15 s), it was shorter (Table 16).

177 Intriguingly, this $13-15$ s response interval corresponds well with the duration that dopamine is

178 detectable in the extracellular space during rewarded trials ${ }^{17}$ (Extended Data Fig. 4d). Both 
rewarded trial types showed higher response peaks at this 'preferred' run duration (Fig. $\mathbf{3 a , b}$ and Extended Data Fig. 4c, top, center panels, Table 17). Calculating the response offset relative to the reward onset in correct trials showed that most responses ended $\sim 10 \mathrm{~s}$ after reward onset, with only a few lasting longer than $15 \mathrm{~s}$ (Fig. 3g). Similarly, aligning the responses to the longest runs (25-40 s) at reward onset showed that their offsets are similar (Fig. 3h). In contrast, for spontaneous runs, the astrocyte response co-varied with run duration (Fig. 3c), with the difference between syncytium response offset and run offset clustering around $0 \mathrm{~s}$, irrespective of run duration (Extended Data Fig. 4b, bottom panel, Table 16). As expected, a histogram of response offsets relative to run offset reflects this high degree of correlation (Fig. 3i). Together, this data suggests that different encoding profiles underlie rewarded and spontaneous runs and that reward-related molecular signals, such as dopamine, modulate astrocytes' run-evoked syncytium responses.

While linear regression analysis is restricted to predefined signal characteristics (e.g., onset, offset, peak, or duration), decoding models can access all information contained within the

194 signals' time course. To infer relevant parameters from the signals' temporal dynamics, we used the k-nearest neighbor $(\mathrm{kNN})$ classifier $^{18}$, one of the most popular supervised machine learning algorithms for time series classification. We trained the classifier on example syncytium traces, represented as vectors in multidimensional feature space with corresponding class labels. In the subsequent test phase, the classifier was tasked with predicting the classes of syncytium transients that the classifier had not used for learning, based on the most frequent class among the $k$ training samples nearest to the query vector. Bayesian optimization was used to select the 201 distance calculation method and $k$, the number of neighbors (Table 18). We visualized classifier 202 predictions using confusion matrices ${ }^{19}$. To evaluate the classifier's performance, we calculated 203 the area under the receiver operating characteristic curve (AUC). This curve captures the true 204 positive versus the false positive rate of the classifier at different classification thresholds, 
205 thereby representing the prediction performance quality irrespective of the chosen threshold.

206 Statistical significance was derived from random permutation testing, shuffling the training data

207 class labels, and calculating the probability that the prediction performance could be explained

208 by chance (Table 19).

209

210 When we used syncytium responses for rewarded and spontaneous trials only, the kNN

211 classifier was able to identify these two classes with high accuracy (85\% correct class

212 assignments; chance level at $50 \%$ ) and $\mathrm{AUC}=0.87$, significantly different from the mean

213 calculated in the permutation test $(A \cup C=0.5$, Fig. 4a). The classifier also confirmed the high

214 predictability of correct and error trials from syncytium responses (86\% accuracy; chance level

215 at 50\%; $A \cup C=0.83$, Fig. 4b). Remarkably, the syncytium response carried information about

216 every trial type, which the classifier could predict from the recorded trials (38\% accuracy;

217 chance level at 20\%) (Extended Data Fig. 5a). Moreover, the classifier predicted the animals'

218 performance level using all traces from all recorded trial types (62\% accuracy; chance level at

$21950 \% ; A U C=0.64$, Extended Data Fig. 5b).

221 Next, motivated by our LME model results showing that astrocyte syncytium responses varied

222 substantially with run duration for spontaneous but not as much for task-related runs, we asked

223 whether the kNN classifier could predict run duration from spontaneous runs (Extended Data

224 Fig. 5c) and task trials (Extended Data Fig. 5d). We found that decoding of run duration was

225 possible from both spontaneous (90\% accuracy; chance level at 33\%) and task trials (46\%

226 accuracy; chance level at $25 \%$ ), with significantly higher accuracy and AUC values when

227 spontaneous trials were used for classification ( $p<0.05$, Kolmogorov-Smirnov test). We

228 reasoned that if the gradual increase of run duration in the defined run duration classes is

229 accompanied by a gradual change in the encoding signal, the classifier decoding performance

230 should be most robust along the main diagonal of the confusion matrix, and confusions between 
231 adjacent classes should be more frequent. To test this idea, we averaged the classification

232 probability along the main diagonal and the parallel diagonals, resulting in the average

233 performance of the classifier as a function of distance from the actual run duration (Extended

234 Data Fig. 5c-d, last panel). The function peaked at the probability for correctly assigning the

235 query traces to their real run duration class, while more erroneous classifications occurred for

236 adjacent run durations. This finding confirms the gradually changing nature of the signal

237 underlying the decoding of spontaneous and task trials and the proper operation of the

238 classifier.

240 While the previous analyses demonstrated that we could decode animal behavior (run context,

241 reward delivery, performance level, run duration) from astrocyte syncytium responses, we also

242 wanted to know whether the responses were relevant for the animals' behavior. If the astrocytic

243 signal is relevant for mouse behavior, the decision should be decodable from correct and error

244 trials. Indeed, we found that the perceptual decision of the animal could be decoded from

245 correct (hit and CR) trials (62\% accuracy; chance level at 50\%; Extended Data Fig. 6a). Next,

246 we trained the kNN classifier on correct decision trials (hit trial: 'yes' decision; CR trial: 'no'

247 decision) and used the signals for erroneous decisions (miss trial: 'no' decision, FA trial: 'yes'

248 decision) as a test dataset. We found that error trials also carried significant information about

249 the decision (Extended Data Fig. 6b). Finally, we examined whether areas M1/M2 encoded

250 information about the nature of the sensory information that was essential for the animals'

251 decision. In accordance with our LME model results (Fig. 2f), the classifier was able to decode

252 information about the presented stimulus intensity from the astrocyte syncytium responses to hit

253 trials (65\% accuracy; chance level at 50\%; Extended Data Fig. 6c). Importantly, decoding of

254 stimulus type information was not possible from astrocyte syncytium responses to miss trials

255 (53\% accuracy; Extended Data Fig. 6d), implying that sensory information important for 
decision-making was absent in error trials. Together, these findings suggest that the information encoded by astrocyte syncytium responses is relevant for animal behavior.

\section{Discussion}

260 In summary, the astrocyte syncytium calcium response is a complex yet deterministic signal

261 encoding several aspects of behavioral context. Signal onset was tightly linked to run onset in

262 rewarded trials, with an earlier calcium response encoding the animal's decision (Fig. 2e) and

263 stimulus intensity (Fig. 2f). Interestingly, in spontaneous runs, the response onset had a

264 significant delay for longer run durations. Response duration was influenced by both decision

265 correctness in task trials and run duration (Fig. 2g). Response offset correlated with dopamine

266 levels in rewarded trials and run offset in spontaneous runs (Extended Data Fig. 4d, Fig. 3i).

267 The overall strength of the calcium response was impacted by trial type, with rewarded trials

268 showing the most notable increase (Fig. 2 g). The amplitude was also significantly modulated by

269 the animal's performance level (Fig. 2i) and potentially by run parameters linked to reward

270 expectation (Fig. 3a-b). The inter-run interval had a significant impact on the probability and

271 strength of the astrocytic response in task trials and spontaneously initiated runs. Notably, the

272 information encoded in the astrocyte syncytium calcium responses was behaviorally relevant

\section{3 (Extended Data Fig. 6).}

275 What mechanisms might control astrocyte syncytium responses? Because astrocytes do not

276 exhibit stereotyped calcium waveforms like those evoked by neuronal action potentials, previous

277 work suggested that their transients result from spatial and temporal integration of behavior-

278 related extracellular molecular signals released, for example, by local and projection neurons ${ }^{1}$.

279 The complex yet deterministic nature of astrocyte syncytium responses revealed by our study

280 supports this notion. Response duration and amplitude depended, amongst others, on run

281 duration, suggesting integration of ongoing synaptic activity by astrocytes (Fig. 3). Rewarded hit 
and CR trials showed larger syncytium responses than unrewarded trials with a 'preferred' run duration. One possible explanation for this 'preferred' run duration is that 13 s-long runs offer

284 the highest reward probability to the animal, with astrocytes reflecting the corresponding local activity of M1/M2 neurons. Another possibility is that dopamine's time course determines the peak of the astrocyte syncytium response in rewarded runs. The time course of previously measured dopamine signals in the same region and task are consistent with this hypothesis ${ }^{17,20}$

(Fig. 2; Extended Data Fig. 4). We also found that astrocyte syncytium responses in run trials are significantly different from no-run trials. Specifically, the probability of miss and CR trials without a run was significantly lower than those with a run (Extended Data Fig. 7a,b). Moreover, the syncytium responses to no-run trials had significantly longer response latencies (Extended Data Fig. 7c), were shorter, reached lower peak values, and showed lower total activation extent (Extended Data Fig. 7d). This observation seems consistent with previous work showing that locomotion mediates noradrenaline release and widespread astrocyte calcium excitation and that the astrocyte response is boosted in the presence of sensorimotorevoked local neural activity ${ }^{11,12}$. How astrocyte syncytium responses may differ in behavioral

297 tasks that do not involve a running response (e.g., lever press/release task) remains to be determined. Apart from dopamine and noradrenaline, additional neuromodulator signals, such as acetylcholine, may also modulate astrocytes' phasic syncytium responses ${ }^{21,22}$. Our finding that astrocyte responses were larger during low-performance states may, at least in part, be explained by higher tonic neuromodulator levels (e.g., noradrenaline) associated with this cortical state ${ }^{23,24}$ (Fig. 2). Together, our data seem consistent with the concept of spatial and

303 temporal integration of neurotransmitter and neuromodulator signals in shaping astrocyte

304 syncytium responses in the M1/M2 cortex. Nevertheless, to better understand the syncytium

305 signal's building blocks and regional differences, an analysis of individual regions of interest or 306 astrocyte compartments may be informative. 
308 How might astrocyte syncytium responses affect local neural circuits? Our findings suggest that 309 astrocytes' signaling is encoding relevant behavioral information (Extended Data Fig. 6).

310 However, one of the striking features of astrocyte syncytium responses is their seconds-long

311 delay relative to run onset (Fig. 2; Extended Data Fig. 1), likely due to signal integration within

312 astrocytes (e.g., IP3, which mediates endoplasmic reticulum (ER) calcium release) ${ }^{3,25}$. This

313 delay, together with the syncytium responses' slow time course, indicates that astrocyte

314 excitation likely serves complementary roles to neuronal activity, particularly those preceding or

315 initiating the behavioral response (e.g., decision making or motor planning). One potential role

316 of astrocyte syncytium responses may be circuit regulation. Following task execution, astrocytes

317 may restore the neural circuit's ionic and transmitter homeostasis, thus ensuring the circuit's

318 continued operation with optimal signal-to-noise ratio and gain. Additionally, they may actively

319 tune the system when the executed behavior does not reliably achieve the desired outcome

320 (e.g., reward $)^{26,27}$. By establishing a computational "review period" of past events, astrocytes

321 could potentially inform future behavior, enabling trial-to-trial behavioral adjustments or learning.

322 If these considerations are correct, they might explain why astrocyte syncytium responses

323 depended on behavioral performance and perceptual level (Figs. 1-2). These hypotheses might

324 be tested by an in-depth analysis of trial history and performance as a function of the inter-run

325 interval, which strongly affects astrocytes' response probability and strength (Extended Data

326 Fig. 2).

327

328 Given the complex yet predictable syncytium responses (Fig. 4; Extended Data Figs. 5-6), it is 329 conceivable that astrocyte calcium excitation mediates more than one output and on multiple

330 timescales. Astrocytes can modulate neural circuit activity on the seconds (i.e., individual trial)

331 timescale by releasing neuroactive substances in a calcium-dependent manner (e.g., 
332 ATP/adenosine, D-serine, potassium) ${ }^{3}$. Neural circuit activity can also be modulated on the

333 minutes (i.e., performance level) timescale by activity-dependent changes in astrocyte

334 transporter activity, gap junctional coupling, metabolic support, or perisynaptic process

335 structure.

337 How might these open questions about astrocyte syncytium responses be addressed? The

338 quantitative visual detection task and computational methods employed in our study may help

339 address these fundamental questions. In conjunction with genetically encoded neurotransmitter

340 and neuromodulator sensors, our standardized approach may help reveal how behavior-

341 dependent extracellular signals relate to astrocyte activity, as exemplified for dopamine

342 (Extended Data Fig. 4). However, this may require further optimization of current transmitter

343 sensors and their color variants to enable concurrent and high-resolution measurement of

344 corresponding transient maps. Simultaneous recording of astrocyte and projection neuron

345 activity can only partly replace such measurements, as calcium spiking does not identify the

346 type and quantity of the transmitter(s) released or its spatial spread. Likewise, new indicators for

347 intracellular signaling (e.g., IP3, cAMP, or PKA) and functional alterations (e.g., proximity

348 assays) may in the future allow measurement of how the various molecular signals are

349 integrated within astrocytes, how this spatiotemporal integration relates to astrocyte syncytium

350 responses, and how these responses modulate astrocyte output ${ }^{28,29}$. One approach to

351 determine the effect of astrocyte syncytium responses on local neural activity may be to

352 leverage their intrinsic properties. We showed that the probability, onset, and magnitude of

353 syncytium responses depend on inter-run distance (Extended Data Fig. 2), an effect previously

354 described for cerebellar astrocytes and likely dependent on ER calcium store dynamics ${ }^{10}$.

355 Animals trained to perform visual detection task trials at various inter-run distances may provide

356 insight into how local neural activity changes in the presence or absence of astrocyte syncytium 
357 responses. However, the dependency of astrocyte syncytium responses on trial-type,

358 performance levels, and other behavioral variables suggests that approaches to globally in- or

359 decrease astrocyte excitation (e.g., by opsin, DREADD, calcium pump, or chelator expression)

360 may only partially mimic astrocytes' varied effects on neural circuits. Finally, applying our visual

361 detection task and computational methods to other (e.g., sensory) brain regions should help

362 determine conserved features of astrocyte encoding and circuit modulation and inform models

363 of how astrocyte signaling may need to be incorporated into systems neuroscience. 


\section{References}

369

370 1. Kastanenka, K. V. et al. A roadmap to integrate astrocytes into Systems Neuroscience.

$371 \quad$ Glia 68, 5-26 (2020).

372 2. Nimmerjahn, A. \& Bergles, D. E. Large-scale recording of astrocyte activity. Curr. Opin.

$373 \quad$ Neurobiol. 32, 95-106 (2015).

374 3. Verkhratsky, A. \& Nedergaard, M. Physiology of Astroglia. Physiological Reviews 98, $375 \quad 239-389(2018)$.

376 4. Bazargani, N. \& Attwell, D. Amines, Astrocytes, and Arousal. Neuron 94, 228-231 (2017).

377 5. Nagai, J. et al. Behaviorally consequential astrocytic regulation of neural circuits. Neuron $378 \quad 109,576-596(2021)$.

379 6. Wang, Y. et al. Accurate quantification of astrocyte and neurotransmitter fluorescence dynamics for single-cell and population-level physiology. Nat Neurosci 22, 1936-1944 (2019).

382 7. Bojarskaite, L. et al. Astrocytic Ca2+ signaling is reduced during sleep and is involved in the regulation of slow wave sleep. Nat Commun 11, 3240-16 (2020).

384 8. Curreli, S., Bonato, J., Romanzi, S., Panzeri, S. \& Fellin, T. Glial place cells: complementary encoding of spatial information in hippocampal astrocytes. bioRxiv (2021). doi:10.1101/2021.07.06.451296

9. Doron, A. et al. Hippocampal Astrocytes Encode Reward Location. bioRxiv (2021). doi:10.1101/2021.07.07.451434

10. Nimmerjahn, A., Mukamel, E. A. \& Schnitzer, M. J. Motor behavior activates Bergmann glial networks. Neuron 62, 400-412 (2009).

391 11. Paukert, M. et al. Norepinephrine controls astroglial responsiveness to local circuit 392 activity. Neuron 82, 1263-1270 (2014).

393 12. Ding, F. et al. a1-Adrenergic receptors mediate coordinated Ca2+ signaling of cortical 
astrocytes in awake, behaving mice. Cell Calcium 54, 387-394 (2013).

13. Green, D. M. \& Swets, J. A. Signal detection theory and psychophysics. (Wiley, 1966).

14. Su, M. et al. Expression specificity of GFAP transgenes. Neurochem. Res. 29, 20752093 (2004).

15. Brenner, M., Kisseberth, W. C., Su, Y., Besnard, F. \& Messing, A. GFAP promoter directs astrocyte-specific expression in transgenic mice. J. Neurosci. 14, 1030-1037 (1994).

16. Ali, F. \& Kwan, A. C. Interpreting in vivo calcium signals from neuronal cell bodies, axons, and dendrites: a review. Neurophotonics 7, 011402 (2020).

17. Patriarchi, T. et al. Ultrafast neuronal imaging of dopamine dynamics with designed genetically encoded sensors. Science 360, (2018).

18. Wu, X. et al. Top 10 algorithms in data mining. Knowl Inf Syst 14, 1-37 (2008).

19. Ting, K. M. in Encyclopedia of Machine Learning and Data Mining 260-260 (Springer, Boston, MA, 2017). doi:10.1007/978-1-4899-7687-1_50

20. Patriarchi, T. et al. Imaging neuromodulators with high spatiotemporal resolution using genetically encoded indicators. Nat Protoc 14, 3471-3505 (2019).

21. Reimer, J. et al. Pupil fluctuations track rapid changes in adrenergic and cholinergic activity in cortex. Nat Commun 7, 13289 (2016). dynamics of dopamine and norepinephrine release in murine cortex. Nat. Methods 11, 1245-1252 (2014).

23. Rho, H.-J., Kim, J.-H. \& Lee, S.-H. Function of Selective Neuromodulatory Projections in the Mammalian Cerebral Cortex: Comparison Between Cholinergic and Noradrenergic Systems. Front Neural Circuits 12, 47 (2018).

24. Avery, M. C., Nitz, D. A., Chiba, A. A. \& Krichmar, J. L. Simulation of cholinergic and noradrenergic modulation of behavior in uncertain environments. Front Comput Neurosci 6, 5 (2012). 
420 25. De Pittà, M. et al. Computational quest for understanding the role of astrocyte signaling in 421 synaptic transmission and plasticity. Front Comput Neurosci 6, 98 (2012).

422 26. Ma, Z., Stork, T., Bergles, D. E. \& Freeman, M. R. Neuromodulators signal through

423 astrocytes to alter neural circuit activity and behaviour. Nature 539, 428-432 (2016).

424 27. Mu, Y. et al. Glia Accumulate Evidence that Actions Are Futile and Suppress

$425 \quad$ Unsuccessful Behavior. Cell 178, 27-43.e19 (2019).

426 28. Oe, Y. et al. Distinct temporal integration of noradrenaline signaling by astrocytic second 427 messengers during vigilance. Nat Commun 11, 471 (2020).

428 29. Octeau, J. C. et al. An Optical Neuron-Astrocyte Proximity Assay at Synaptic Distance $429 \quad$ Scales. Neuron 98, 49-66.e9 (2018). 
a

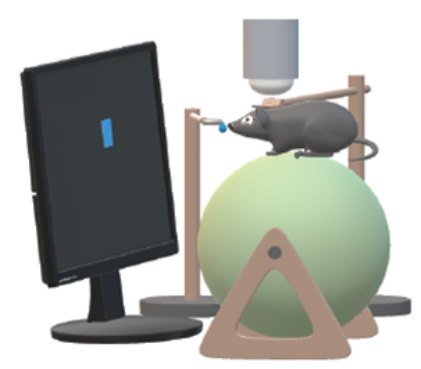

b

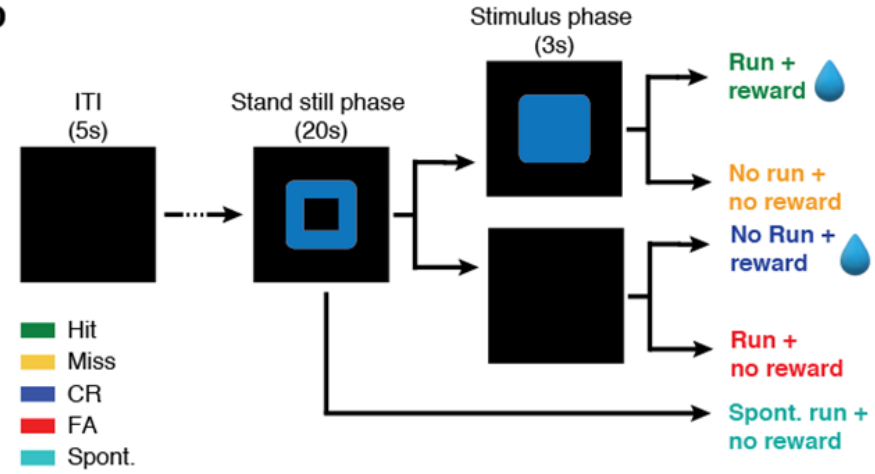

c

\begin{tabular}{|c|c|c|}
\hline & \multicolumn{2}{|c|}{ Stimulus } \\
\hline & present & absent \\
\hline yes & hit & $\begin{array}{c}\text { FA-false } \\
\text { alarm }\end{array}$ \\
\hline no & miss & $\begin{array}{l}\text { CR-correc } \\
\text { rejection }\end{array}$ \\
\hline
\end{tabular}

d

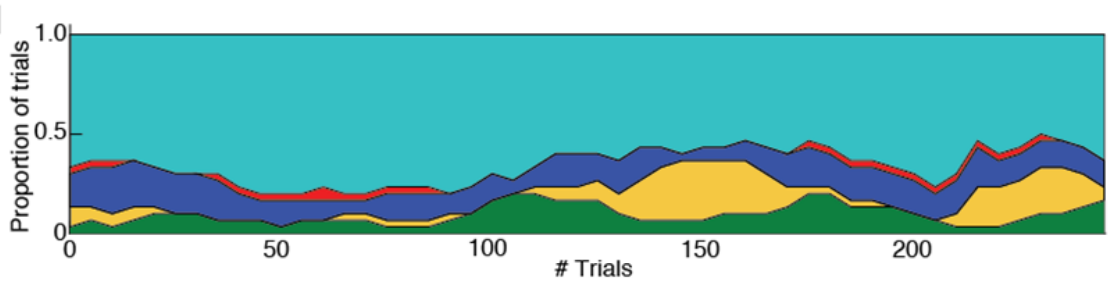

e

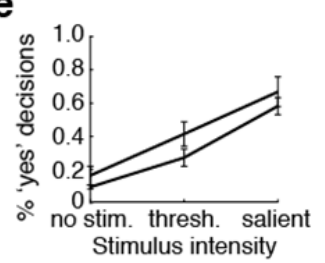

f

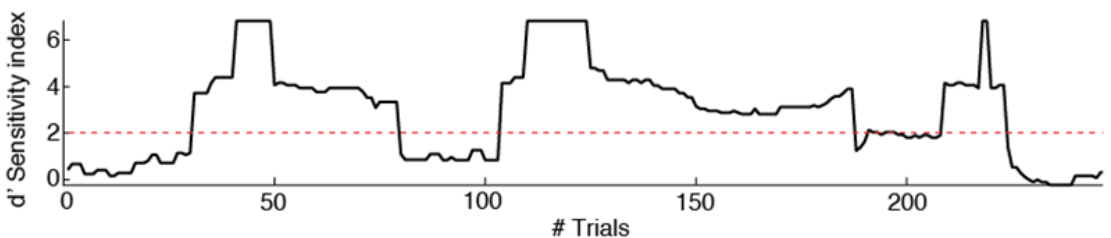

g

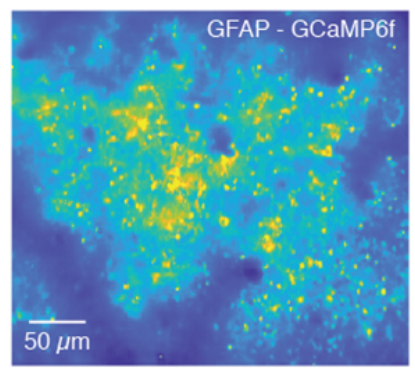

h

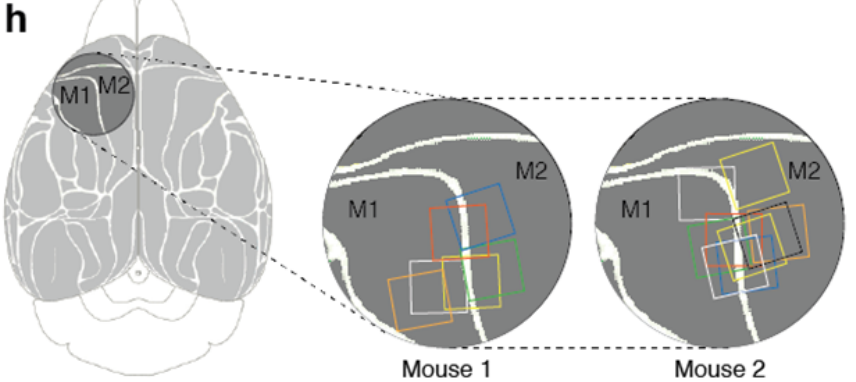

Fig. 1 | Approach for relating astrocyte syncytium calcium signals to behavioral context

434 using detection task variables.

435 a, Schematic of the experimental setup. Head-fixed mice were placed on a spherical treadmill

436 viewing a computer screen. Astrocytic calcium excitation was recorded in layer 2/3 of the M1/M2

437 motor cortex using two-photon microscopy while the mice performed the visual detection task.

438 In total, we recorded 4,837 trials during 21 behavioral sessions (see Methods). b, Schematic of

439 the behavioral protocol. A trial started when mice stopped running for $1 \mathrm{~s}$. A visual cue (blue 
440 frame) instructed mice to remain still for $20 \mathrm{~s}$. Following this stand-still phase, a stimulus (blue

441 square) was presented for $3 \mathrm{~s}$ in $50 \%$ of the trials. In the other half of the trials, no stimulus was

442 shown. Stimulus intensity varied between two levels: salient and close to the perceptual

443 threshold (see Methods). Stimulus presence and intensity were randomly selected. In trials with

444 stimulus presentation, mice were required to start running within the $3 \mathrm{~s}$ stimulus phase to

445 receive a fluid reward. In trials without stimulus presentation, mice had to remain still for a $3 \mathrm{~s}$

446 period to receive the reward. Spontaneous runs during the $20 \mathrm{~s}$ stand-still phase aborted

447 stimulus presentation. Mice were able to initiate a new trial after a 5 s inter-trial interval.

448 c, Signal detection theory classes for behavioral outcomes (hit, miss, correct rejection, and false

449 alarms), given two stimulus conditions (stimulus present or absent) and two possible decisions

450 ('yes, stimulus present' and 'no, stimulus absent'). d, Proportions of behavioral outcomes

451 during one example session. e, Average psychometric detection curves for two representative

452 mice. f, The mouse's performance levels during the example session shown in $d$. The

453 performance level was quantified using the d'-sensitivity index, calculated as the difference of z-

454 scores for 'hit' and 'false alarm' rates. A d'-value of 2 was chosen to distinguish between high-

455 and low-performance states. g, Heatmap of average GCaMP6f fluorescence in layer 2/3 from

456 an example recording in area M1. h, Left, dorsal view of the mouse cortex with the chronic

457 cranial window location indicated (circle). Center and right, imaging locations (squares) within

458 the cranial window for two representative mice. M1, primary motor cortex; M2, secondary motor 459 cortex. 
bioRxiv preprint doi: https://doi.org/10.1101/2021.10.09.463784; this version posted October 10,2021 . The copyright holder for this preprint
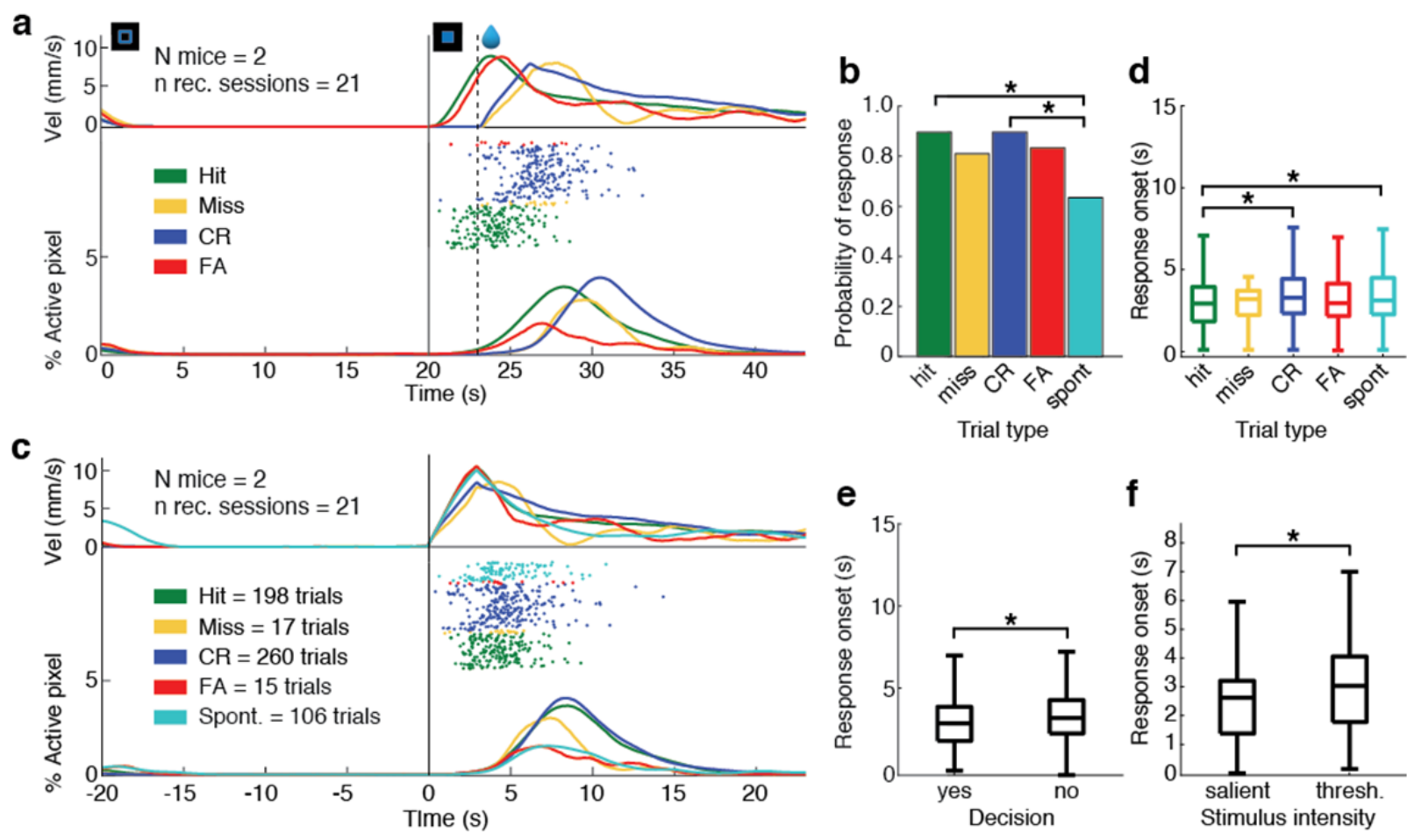

Response Duration

Response Peak

Response Extent

Mean Pixel Activity Duration
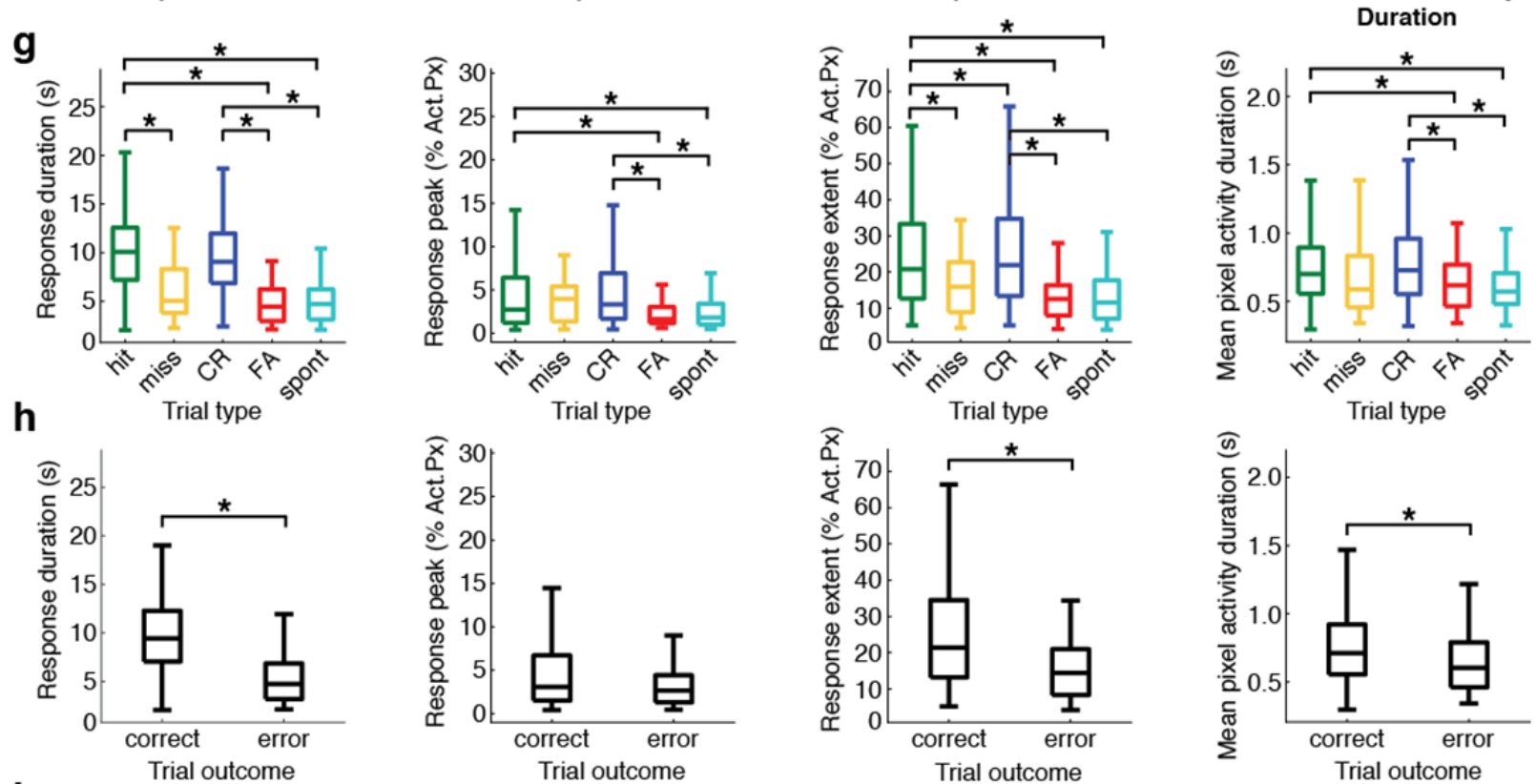

i

Trial outcome
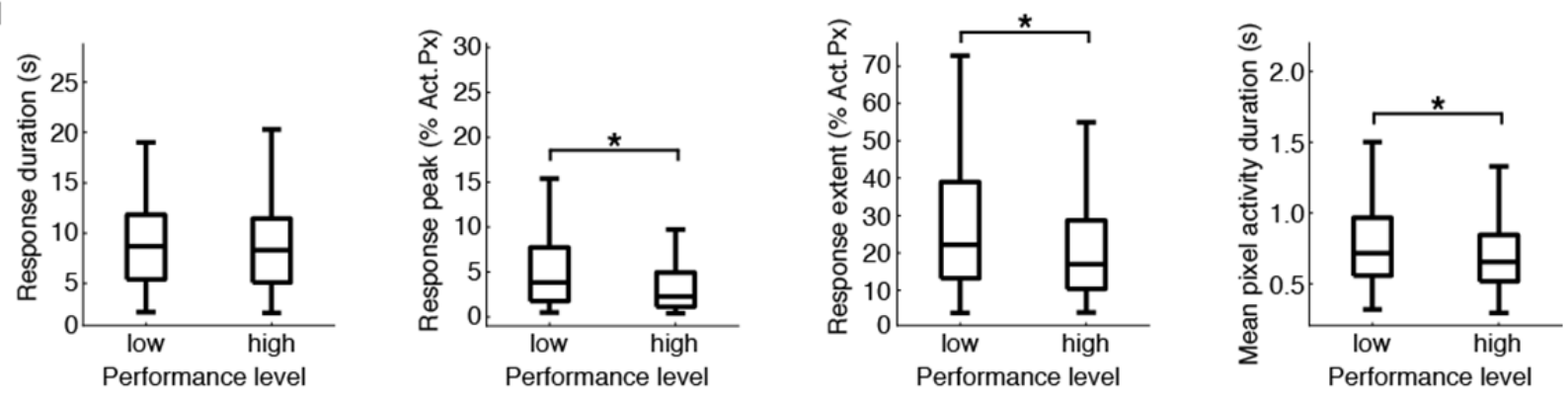
Fig. 2 | Astrocyte syncytium responses encode detection task variables.

a-i, Astrocyte syncytium responses encode reward, the animal's decision (stimulus present or stimulus absent), and performance level. a, Population data showing the astrocyte syncytium

464 signals' dependence on the trial type. Top, running velocity profile for hit (green), miss (yellow), CR (blue), and FA trials (red). Center, onsets (colored dots) for individual qualifying astrocyte syncytium signals by trial type. Bottom, average astrocyte syncytium calcium signals,

467 represented as the percentage of ROA (Regions of Activity) pixels over time (see Extended

468 Data Fig. 1). Each colored trace is an average across the individual trials of a given type 469 aligned to the stand-still cue onset (198 hit, 17 miss, 260 CR, 15 FA trials, and 106 spontaneous 470 runs from 21 recording sessions). Only trials that included a run within a defined parameter 471 range were included to ensure comparability (see Methods). Vertical lines at $20 \mathrm{~s}$ and $23 \mathrm{~s}$

472 mark the stimulus phase. b, Probability of observing a significant astrocyte syncytium response

473 for the different detection task trial outcomes and spontaneous runs. Only spontaneous runs

474 that occurred $15 \mathrm{~s}$ after stimulus onset and before the end of the $20 \mathrm{~s}$ stand-still phase were

475 included in the analysis. c, Same population data as in a, but aligned at run onset (0 s).

476 d, Astrocyte syncytium response onsets relative to run onset for the different trial types. The

477 boxplot marks the median and the 25th and 75th percentiles of the data for each trial type. The

478 whiskers cover $\sim 99.3 \%$ of the data. e, The animals' 'yes' decision (based on hit and FA trials)

479 was encoded by an earlier onset of the astrocyte syncytium response. f, Stimulus intensity was 480 encoded by astrocytes' syncytium response onsets. g, Astrocyte signal strength, as quantified

481 by response duration, peak, the total area under the response curve, and mean pixel activity 482 duration (from left to right), was significantly larger for rewarded than spontaneous and in some 483 characteristics for error trials. $h$, Encoding of rewarded versus error trials. Same layout as in $g$. 484 Rewarded trials showed significantly longer response durations. i, The animal's performance 485 level was encoded primarily by the astrocyte syncytium response amplitude. Low-performance 
bioRxiv preprint doi: https://doi.org/10.1101/2021.10 09.463784; this version posted October 10, 2021. The copyright holder for this preprint (which was not certified by peer review) is the author/funder, who has granted bioRxiv a license to display the preprint in perpetuity. It is made available under aCC-BY-ND 4.0 International license.

486 periods were associated with higher amplitudes. The layout is the same as in $\mathrm{g}$. Statistical

487 significance was derived from linear mixed-effects model (LME) analysis for all comparisons

488 (see Methods, Tables 2-10).

489

490

491

492 


\section{a}

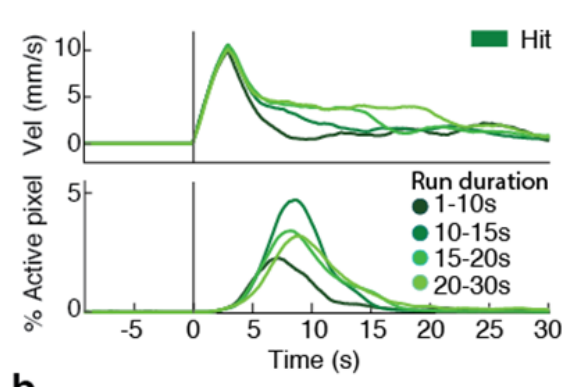

b

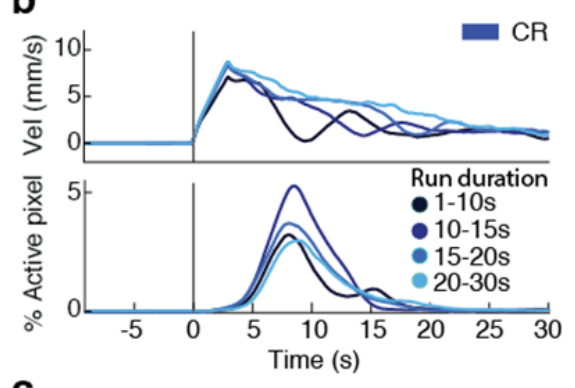

C
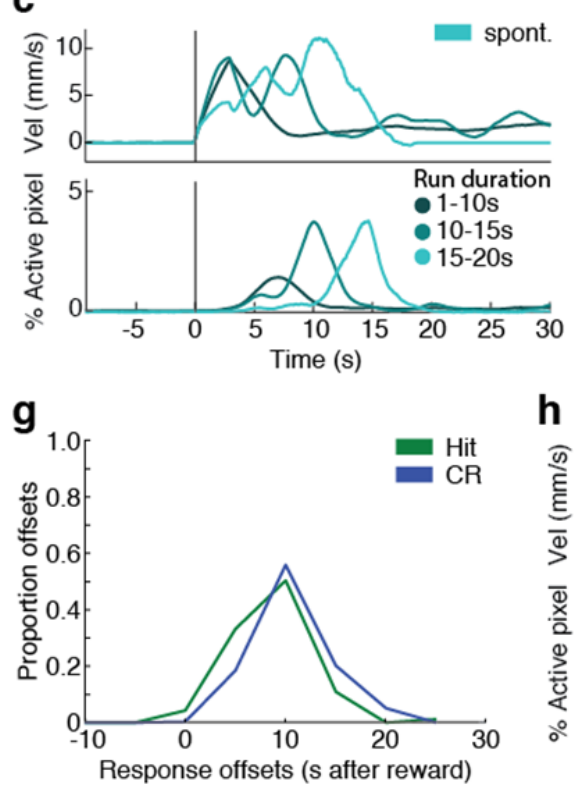
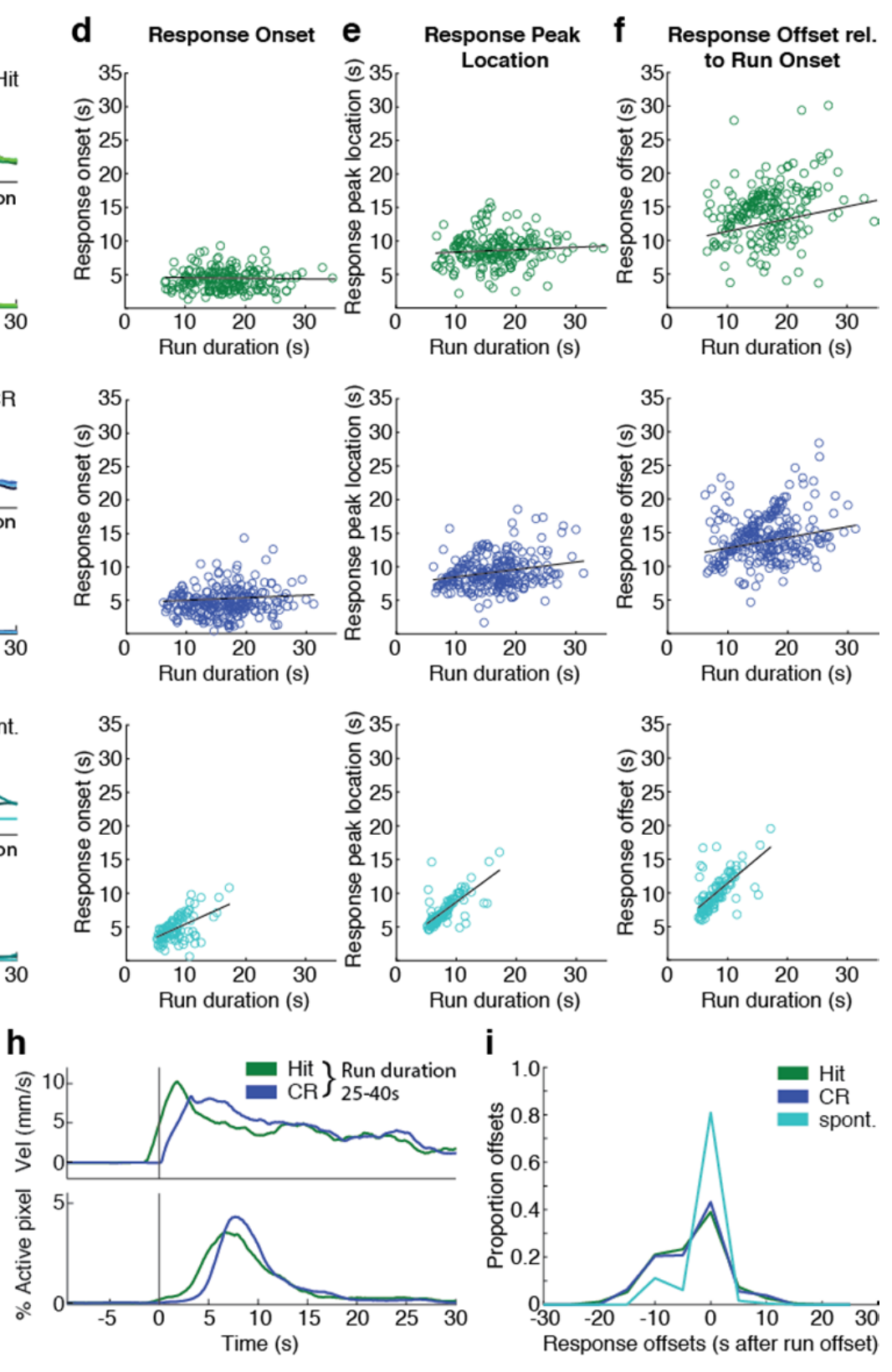

494 Fig. 3| Astrocyte syncytium responses show different run encoding mechanisms.

a-c, Astrocyte syncytium responses, grouped by different run durations, revealed different response profiles for rewarded run trials than spontaneous runs. a, Response profile for hit

497 trials. Top, running velocity profiles. Bottom, astrocyte syncytium responses for hit trials of

498 different run duration $(19,56,75$, and 47 trials of $1-10 \mathrm{~s}, 10-15 \mathrm{~s}, 15-20 \mathrm{~s}$, and $20-30 \mathrm{~s}$ run

499 duration, respectively, from 21 recording sessions). b, Response profile for CR trials. Same 
500 layout as in $a$. The data is an average across $36,73,98$, and 53 runs of $1-10 \mathrm{~s}, 10-15 \mathrm{~s}, 15-20$

501 s, and 20-30 s duration, respectively, from 21 recording sessions. c, Response profile for

502 spontaneous runs. Same layout as in a. The data is an average across 104, 19, and 3 runs of 1 -

$50310 \mathrm{~s}, 10-15 \mathrm{~s}$, and 15-20 s duration, respectively, from 21 recording sessions. d, Astrocyte

504 syncytium response onsets as a function of run duration for hit trials (top), CR trials (center),

505 and spontaneous runs (bottom). e, Peak location of the astrocyte syncytium response as a

506 function of run duration. Same layout as in $d . \mathbf{f}$, Astrocyte syncytium response offsets relative to

507 run onsets as a function of run duration. The layout is the same as in $d . \mathbf{g}$, Histogram of

508 astrocyte syncytium response offsets for rewarded trials (hit and CR) relative to reward onset

509 (see also Extended Data Fig. 4d). Event frequencies were bin-normalized for each run duration

510 interval. h, Profile of astrocyte syncytium responses for the longest runs (25-40 s). Top, running

511 velocity. Bottom, astrocyte syncytium responses aligned at reward onset. i, Histogram of

512 astrocyte response offsets relative to run offsets for hit trials, CR trials, and spontaneous runs.

513 Event frequencies were bin-normalized for each run duration interval. 


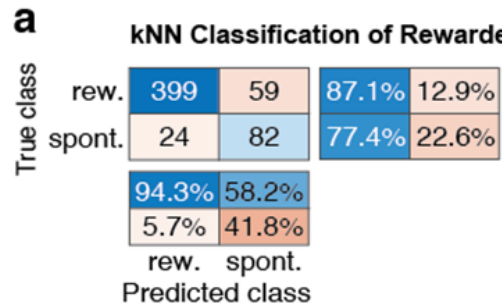

b

Fig. 4 | Detection task variables can be decoded from astrocytic syncytium responses using machine learning approaches.

a-b, The k-nearest neighbor (kNN) classifier allows reliable decoding of rewarded/correct trials

523 from astrocytes' syncytium calcium responses. a, Classifier decoding performance of rewarded

524 trials from rewarded trial and spontaneous run astrocyte syncytium responses. Left, classifier

525 confusion matrices with rows representing the true classes and columns showing the classifier

526 predictions. The main diagonal shows how frequently the classifier correctly assigned the trials

527 to their real category (accuracy). Off-diagonal cells correspond to the count of incorrectly

528 classified trials. A row-normalized row summary and a column-normalized column summary

529 display the percentages of correctly and incorrectly classified trials for each true class or

530 predicted class, respectively. Center, receiver-operating characteristic (ROC) curve and area

531 under the ROC curve (AUC) for the classifier's output. Right, true data mean AUC values (black)

532 were obtained using a 10 -fold cross-validation design, repeated 100 times, and compared to the

533 mean AUC values from shuffled trials (gray) when syncytium responses were randomly 
bioRxiv preprint doi: https://doi.org/10.1101/2021.10.09.463784; this version posted October 10,2021. The copyright holder for this preprint (which was not certified by peer review) is the author/funder, who has granted bioRxiv a license to display the preprint in perpetuity. It is made available under aCC-BY-ND 4.0 International license.

534 assigned to one of the two classes. b, Classifier decoding performance of rewarded trials from

535 rewarded and erroneous trial syncytium responses. The layout is the same as in a. Error bars

536 indicate s.e.m.

537 


\section{Methods}

\section{Experimental model and subject details}

All procedures were performed following the National Institutes of Health $(\mathrm{NIH})$ guidelines for

543 the Care and Use of Laboratory Animals and were approved by the Institutional Animal Care

544 and Use Committee (IACUC) at the Salk Institute. Mouse strains used in this study included

545 Gfap-Cre 73.12 (RRID: IMSR_JAX:012886) and Ai95D mice (RRID: IMSR_JAX:024105) ${ }^{30,31}$.

546 All imaging and behavioral experiments involved heterozygous male mice $(\mathrm{N}=4)$. Mice

547 underwent two surgeries: head plate implantation at 8-10 weeks of age and cranial window

548 implantation at $\sim 12$ weeks of age. Training started $\sim 7$ days after each surgery. Mice were water-

549 restricted to $25 \mathrm{ml} \mathrm{kg}^{-1}$ per day and maintained at $80-85 \%$ of their normal ad-libitum weight

550 during training and imaging. Optical recordings were performed at $\sim 20$ weeks of age. Of the five

551 mice trained on the task, one failed to reach proficiency. Mice were typically group-housed,

552 provided with bedding and nesting material, and maintained on a $12 \mathrm{~h}$ light-dark cycle in a

553 temperature (around $22^{\circ} \mathrm{C}$ ) and humidity controlled (45-65\%) environment. The animals had ad

554 libitum access to standard rodent chow and water outside of training and imaging periods.

Live animal preparation

558 Head plate and cranial window implantation were performed as previously described ${ }^{17,20}$. Briefly,

559 mice were anesthetized with isoflurane (4\% and $2 \%$ for induction and maintenance,

560 respectively) on a custom surgical bed (Thorlabs Inc., Newton, NJ). Body temperature was

561 maintained at $36-37^{\circ} \mathrm{C}$ with a DC temperature control system. Ophthalmic ointment was used to

562 prevent eyes from drying. The skin at the surgical site was cleaned and disinfected with $70 \%$

563 ethanol and Betadine. A small $(\sim 10 \mathrm{~mm})$ incision was performed along the midline. The scalp

564 was pulled open, and the periosteum was cleaned. A portion of the scalp was surgically

565 removed to expose frontal, parietal, and interparietal skull segments. A custom metal plate was 
affixed to the bone above the motor cortex with C\&B Metabond Quick Adhesive Cement (Parkell

567 Inc., Edgewood, NY). The cement also covered all other exposed skull regions.

After initial training, a custom-made cranial window was implanted to enable chronic two-photon

570 imaging ${ }^{32}$. The skull was thinned above the motor cortex, and a craniotomy was performed (2.5

$571 \mathrm{~mm}$ diameter; centered around AP $1.5 \mathrm{~mm} / \mathrm{ML} 1.5 \mathrm{~mm}$ ). The dura mater was kept intact. The

572 craniotomy was sealed with a custom three-layered cover glass assembly (each No.1 thickness)

573 with the two layers closest to the cortex consisting of two circular $2.5 \mathrm{~mm}$-diameter coverslips

574 and the outermost layer consisting of a circular $3 \mathrm{~mm}$-diameter cover glass that rested on the

575 thinned skull. UV-curing optical adhesive (NOA 71, Norland Products, Inc.; cat. no. 7106) was

576 used to attach the coverslips one at a time, taking care to avoid air inclusions that might

577 interfere with imaging or facilitate cover glass detachment during the implantation period.

578

579

580

581

582

583

584

585

586

587

588

589

590

591

592

\section{Behavioral setup and data acquisition}

Animal training was performed in a sound-attenuating cubicle (ENV-017M, Med Associates Inc.) using a custom-built setup. This setup included a color LCD monitor for stimulus presentation (12.1" LCD Display Kit/500cd/VGA, ICP Deutschland GmbH). Noise in optical recordings was minimized by covering the monitor with a color filter (R342 Rose Pink, Rosco Laboratories Inc.). The setup also included a spherical treadmill (Habitrail Mini Exercise Ball, Animal World Network), allowing the animal to run freely or when instructed. Mice were placed on the treadmill facing the LCD display. Head fixation was achieved by clamping the head plate with custombuild holders. An optical encoder (E7P OEM, US Digital) attached to the treadmill enabled measurement of both speed and direction of ball movement. Water reward was delivered with a programmable syringe pump (NE-500 OEM Syringe Pump, New Era Pump Systems, Inc.).

Behavior-related signals were acquired through a data acquisition board (PCl-6221, National Instruments) connected to a breakout box (BNC-2110, National Instruments) and interfaced to 

MATLAB-based software MonkeyLogic (www.monkeylogic.net) ${ }^{33,34}$ controlled the behavioral

595 task sequence. Custom-written functions were added to MonkeyLogic to enable analysis and

596 control of ball rotation parameters. Treadmill encoder signals and trial marker codes, generated

597 by MonkeyLogic, were acquired ( $10 \mathrm{kHz}$ sampling rate; $\pm 5 \mathrm{~V}$ input range) in sync with the

598 imaging data. Simultaneous acquisition through the microscope's software (MScan; Sutter

599 Instrument Company) allowed run parameters, behavioral task events, and image frames to be 600 linked with high temporal precision.

Animal training

603

Mice were handled/tamed on two consecutive days before behavioral training to reduce stress.

605 During the first two training days, mice spent $\sim 15-30 \mathrm{~min} /$ day in the setup to become

606 accustomed to head restraint. Mice were then trained daily for $60-90$ min during which they

607 performed $\sim 300-700$ trials. A sequence of trial task events was initiated when mice stood still on

608 the ball for $1 \mathrm{~s}$. First, a blue square frame was displayed on the monitor, requiring the animal to

609 continue standing still for $20 \mathrm{~s}$ (ball rotational velocity $\leq 2 \mathrm{~mm} / \mathrm{s}$ ). If the mouse remained still for

610 this entire stand-still phase, a second stimulus (filled blue square) was presented for $3 \mathrm{~s}$ in $50 \%$

611 of trials, instructing the mouse to initiate a run. The stimulus was presented at two intensities:

612 salient or close to the perceptual threshold (determined empirically towards the end of the

613 training and kept at the same level during recordings). If the mouse initiated sustained

614 movement during the $3 \mathrm{~s}$ stimulus phase (ball rotational velocity $>10 \mathrm{~mm} / \mathrm{s}$ for at least $1 \mathrm{~s}$ ), a

615 water reward was delivered (hit trial). If no running occurred, the trial counted as a miss trial. In

616 the $50 \%$ of trials where no stimulus was presented, the mouse received a fluid reward when it

617 remained still on the ball for $3 \mathrm{~s}$ (correct rejection, CR). Running during the $3 \mathrm{~s}$ period was

618 counted as a false alarm (FA) trial. The trial was aborted and counted as a spontaneous run if 
619 the animal moved during the $20 \mathrm{~s}$ stand-still phase (ball rotational velocity $>2 \mathrm{~mm} / \mathrm{s}$ ). The mouse

620 could initiate a new trial after an inter-trial interval (ITI) of $5 \mathrm{~s}$.

622 In vivo two-photon imaging

624 Once mice had reached proficiency on the task, two-photon imaging commenced. Mice were

625 imaged daily for 9-12 days while performing the task. We used a resonant scanning two-photon

626 microscope (Sutter Instrument) equipped with a pulsed femtosecond Ti:Sapphire laser

627 (Chameleon Ultra II, Coherent) for simultaneous optical and analog data acquisition. GCaMP6f

628 fluorescence was excited with $910 \mathrm{~nm}$ light and detected using an ET525/70M emission filter

629 (Chroma Technology Corp.) and H7422-40 GaAsP photomultiplier tube (Hamamatsu

630 Photonics). Average excitation power depended on imaging depth (typically 55-66 mW). The

631 typical recording depth was 100-135 $\mu$ m below the pia. Data were acquired using a Nikon

632 16×0.8-NA water immersion objective. A custom-made blackout curtain around the

633 microscope's detector was used to reduce light contamination by the LCD monitor. Images

$634(512 \times 512$ pixels $)$ were acquired at 1.0x Zoom $(\sim 510 \times 640 \mu \mathrm{m}$ effective field of view after

635 cropping) and $\sim 30.9$ frames/s using MScan software (Sutter Instrument Company). Each

636 recording session consisted of five to twelve $\sim 10$ min recordings, separated by short imaging

637 breaks (3-5 $\mathrm{min}$ ). Recordings within a given session were performed at the same location to

638 maximize the number of trial repetitions for analysis. Recordings from different sessions on

639 consecutive days were offset either laterally or axially to maximize the tissue volume being

640 sampled (Fig. 1h).

644 All data analyses were performed using custom-written MATLAB scripts (The MathWorks Inc).

645 The encoder signal (frequency of voltage changes) was converted to run velocity and smoothed 
646 with a 3 s moving average window. This smoothing widened the signal by half the window size.

647 We, therefore, shifted the smoothed velocity trace by $+1.5 \mathrm{~s}$ for all analyses involving

648 alignments at task events (trial onset, reward onset). Smoothing also lowered peak running

649 speeds (Fig. 2a). Run onset threshold was set at $0.5 \mathrm{~mm} / \mathrm{s}$, while run offset was defined as the

650 time when running speed fell below $0.1 \mathrm{~mm} / \mathrm{s}$. We chose these low thresholds because even

651 small movements could elicit calcium responses. Run onsets $<0.5 \mathrm{~s}$ after the offset of a

652 previous run were considered as one running event. A trial-associated run counted as hit or FA

653 if running lasted $>1 \mathrm{~s}$ and exceeded $30 \mathrm{~mm} / \mathrm{s}$. The absence of ball movement $(<0.5 \mathrm{~mm} / \mathrm{s})$

654 during the stimulus phase counted as CR trial. Run velocity was measured in real-time during

655 animal behavior (without temporal smoothing). While all hit and FA trials included a run during

656 the stimulus phase, miss and CR trials did not exhibit a run during this phase. However, they

657 were often followed by a run after stimulus offset or reward delivery, respectively.

659 To quantify the animals' task performance, we recorded all trial outcomes (Fig. 1c) and reaction

660 times (RTs) (the time interval between stimulus and run onset). Mice were considered to have

661 reached task proficiency when the proportion of correct decisions (hit and CR trials) exceeded

$66250 \%$ over a 50 trial performance interval. Additionally, RTs for correct 'yes' decisions (hit trials)

663 had to drop below $1.5 \mathrm{~s}$. The psychometric curve for each mouse (Fig. 1e) was computed

664 based on the proportion of 'yes' decisions for stimulus-absent (FA), threshold, and salient

665 stimulus intensity trials (hit trials). A steep increase in miss trials at the end of the session

666 indicated that mice had lost interest in the water reward. Trials beyond that point were excluded

667 from the average performance analysis. To quantify the level of performance throughout the

668 session, we calculated the discriminability index d-prime (d', Fig. 1f) for each session as

$669 Z($ hit/(hit + miss $))-Z(F A /(F A+C R))$, with $Z(p), p \in[0,1]^{13}$. All trials during d'>2 phases were

670 considered high-level performance trials. Trials during d' $<2$ phases were deemed to be low-level

671 performance trials. 


\section{Image data processing and analysis}

675 Lateral image motion (e.g., due to mouse movement) was corrected using the non-rigid

676 movement correction algorithm NoRMCorre ${ }^{35}$. We used 200 frames from the first recording of a

677 given session to compute the registration template. The same reference image was used to

678 correct the image motion of other recordings taken at the same location.

680 We excluded regions over and immediately surrounding blood vessels to reduce artifacts

681 caused by vascular dilation and constriction. First, we calculated a baseline image by smoothing

682 the image data temporally with a moving average of $1 \mathrm{~s}$. Next, we determined the mode of the

683 pixels. Areas below the $70^{\text {th }}$ percentile of the baseline image's pixel intensity distribution were

684 automatically excluded from data analysis.

686 While the GFAP promoter drives expression in most and predominantly astrocytes, a limited 687 region-dependent neuronal expression $\left(0.5-5 \%\right.$ of labeled cells) has been described ${ }^{14,36}$. To 688 identify corresponding regions in our data, we first calculated the mean intensity projection of all 689 recordings at a given imaging site and segmented this image using the CellProfiler image690 analysis software ${ }^{37}$. We allowed the total area of segments to vary between 8 and 300 pixels.

691 Next, we extracted the fluorescence time trace $F(t)$ from all segments by averaging the pixel 692 intensities of all pixels within individual segments. $\Delta \mathrm{F}(\mathrm{t}) / \mathrm{F}$ was calculated as $(\mathrm{F}(\mathrm{t})-$

693 mean F) / mean F. Segments were classified manually by considering their morphology (from

694 the mean intensity projection image), waveform shape, and event frequency and pattern (from

695 the corresponding $\Delta \mathrm{F}(\mathrm{t}) / \mathrm{F}$ trace). Segments showing features of neuronal activity ${ }^{16}$ were

696 excluded. Between $4.5 \%$ and $12.8 \%$ of segments displayed neuronal characteristics in areas

697 M1/M2. 
699 To capture the high spatiotemporal complexity of astrocytes' calcium signals, we implemented a

700 previously described activity-based algorithm based on Regions-of-Activity (ROA) analysis ${ }^{7}$ with

701 a few modifications. The data were smoothed with a Gaussian filter ( $\sigma=3$ pixels). To remove

702 slow drifts in the calcium baseline, we detrended the time course of each pixel using the

703 MATLAB function detrend() instead of bandpass filtering the data. Fluorescence events were

704 determined based on noise-based thresholding over time for each pixel. First, the signals were

705 high pass filtered. Then, the standard deviation of each pixel's noise over time was calculated.

706 Whenever a given pixel's value in the standard deviation image exceeded the corresponding

707 value 5-fold, the pixel was considered active. The syncytium response signal was calculated as

708 the sum of the active pixels in the field of view (FOV) normalized by the total GCaMP6f labeled

709 area over time.

711 Each astrocyte syncytium time trace includes multiple repetitions of the same trial type. To

712 characterize the syncytium response to a given trial, we quantified its temporal features, such as

713 response onset/offset, probability, and strength. To calculate response onset/offset, we first

714 determined the mean syncytium response distribution during the baseline period (7-2 s before

715 stimulus onset). We defined the $95^{\text {th }}$ percentile of this baseline activity distribution as the

716 significant response threshold (Extended Data Fig. 1b). Time points at which the signal

717 surpassed or fell below this threshold relative to run onset were defined as response onset and

718 offset, respectively. Response probability was calculated by determining the proportion of trials

719 during which the astrocyte syncytium signal exceeded the threshold value during the $0.5-15 \mathrm{~s}$

720 response interval after run onset (if a run happened) or $0.5-17 \mathrm{~s}$ after stimulus onset (if no run

721 was detected during that trial). Response strength was characterized by the (1) response

722 duration (i.e., the interval between response on- and offset), (2) response peak (the maximum

723 value reached during the response duration), (3) total spatial extent of the syncytium response 
724 (defined as the percentage of active pixels in the projection image during the response interval),

725 and (4) mean duration of consecutively active pixels during the response interval.

$727 \quad$ Statistical analysis

In total, we analyzed 4,837 trials from 21 behavioral sessions. Only trials with $>7 \mathrm{~mm} / \mathrm{s}$ ball

730 rotational velocity (smoothed) and $>5$ s run duration were included in our analysis to ensure trial

731 comparability. The analysis also included only spontaneous runs starting $>15 \mathrm{~s}$ after the stand-

732 still cue onset to ensure comparability of task-trial and spontaneous run-evoked syncytium

733 responses. The resulting numbers of qualifying trials are shown in Table 1. Qualifying trial

734 traces from all animals, all sessions, and trial types, associated with a run and significant

735 syncytium response, were averaged and aligned at trial onset (Fig. 2a) or run onset to calculate

736 population responses (Fig. 2c).

738 To quantify the relationship between astrocyte syncytium responses and behavioral variables,

739 we performed linear mixed-effects analyses in MATLAB ${ }^{38}$ (Fig. 2). Separate encoding models

740 were fitted for astrocyte syncytium response probability, onset, duration, peak (log-transformed),

741 total extent (log-transformed), and the mean duration of pixel activation (log-transformed) as

742 dependent variables. Subject (mouse identity), recording area (M1/M2), trial type (hit, miss, CR,

743 FA, spontaneous run), performance level (high/low), current run duration, current run amplitude,

744 preceding run duration, preceding run amplitude, and the interval between current run onset and

745 preceding run offset were included as fixed effects in the model. The recording session was

746 treated as a random effect. To decide which behavioral variables to include in the model, we

747 first performed a univariate analysis. We included the fixed factors separately and added the

748 random effects to the model. If the $p$-value of a dependent variable's relationship to the tested

749 fixed effect was $<0.1$, the factor was considered for inclusion in the final model. Next, a model 
750 with all qualifying fixed effects and the random effect was set up for each dependent variable,

751 followed by a backward step-down model selection. With every iteration, we excluded the fixed

752 effect with the highest non-significant $p$-value until the $p$-values of all remaining factors were

$753<0.05$. This value was chosen as the significance criterion. Visual inspection of residual plots for

754 the final models did not reveal any apparent deviations from homoscedasticity or normality. We

755 fitted a binomial generalized linear model using the MATLAB function fitglme() (Table 2) to

756 analyze the relationship between syncytium response probability and behavioral parameters.

757 We included only trials followed by a run and compared the proportions of trials with significant

758 syncytium response to the proportion of trials without one (i.e., when the syncytium response

759 remained under the threshold value). We also included the interaction between trial type and

760 run duration in the model because this described our data better $(p<0.0001$, Likelihood ratio

761 test). For all other dependent variables, we fitted ordinary linear mixed-effects models using the

762 MATLAB function fitlme() and included only trials with a run and significant syncytium response

763 (Tables 3-10). To analyze differences between trials with or without a run, we used all trials with

764 a significant syncytium response aligned at trial onset (Table 11). Mixed-effects model

765 parameters were estimated by the maximum likelihood method. The significance of the

766 regression coefficients was assessed using the t-statistic.

768 To investigate the relationship between run duration and syncytium response duration

769 (Extended Data Fig. 4a), onset, peak location, and offset, aligned on run onset (Fig. 3d-f) or

770 run offset (Extended Data Fig. 4b), we also applied linear regression analysis. Linear mixed-

771 effects models were fitted separately for the rewarded trials (hits, CR) and spontaneous runs.

772 Model selection criteria and analysis were the same as described above. The percent slope was

773 determined by multiplying the regression coefficient by 100 (Tables 12-16). 
775 To examine the relationship between response peak and run duration, we used responses with

776 peak values $>3 \%$ active pixels (Extended Data Fig. 4c). The peak values appeared to reach

777 their maximum for 13 s-long runs. Linear models were fitted to trials with shorter $(<13 \mathrm{~s})$ and

778 longer run durations (>13 s) separately for rewarded trials and spontaneous runs (Table 17).

779

780 To determine what triggers astrocyte syncytium response offset, we plotted the distribution of

781 rewarded trial offsets aligned at reward onset (Fig. 3g). For comparison, we also plotted the

782 rewarded and spontaneous run offsets aligned at run offset (Fig. 3i). For both histograms, we

783 normalized the distribution of each predefined run duration interval (5-10 s, 10-15 s, 15-20 s, 20-

$78425 \mathrm{~s}, 25 \mathrm{~s}$-maximum run duration). For final representation, the normalized distributions were

785 averaged. This approach helped to avoid biasing run durations that appeared more frequently

786 during trials.

788 To decode information from astrocyte syncytium responses, we applied the k-Nearest Neighbor 789 (kNN) classifier using the MATLAB function fitcknn() (Fig. 4 and Extended Data Figs. 5-6). We

790 represented the syncytium responses (\% active pixels over time), from run onset to $30 \mathrm{~s}$ after

791 run onset, as vectors in multidimensional feature space. The prior probabilities for all classes

792 were defined as equal (i.e., $1 /$ number of classes). The classifications were performed in a $10-$

793 fold cross-validation design (i.e., data was partitioned in 10 randomly chosen subsets). One

794 data subset was used to validate the model, while the remaining subsets were used for training.

795 We used automatic hyperparameter optimization to find hyperparameters that minimized the 10-

796 fold cross-validation loss (see Table 18 for resulting parameters for each classification).

797 Accuracy was calculated as the number of correct predictions divided by the total number of

798 predictions. For decoding the animal's decision from erroneous syncytium responses, the

799 classifier was trained on correct responses only. It was then tested on error trials that the

800 classifier had not seen before. 
802 To visualize the classifier performance, we used the "Receiver Operator Characteristic" (ROC)

803 curve. We calculated the "Area Under the Curve" (AUC) to measure the classifier's ability to

804 distinguish between classes. We extended the ROC-AUC calculation to multiclass classification

805 decoding, using the "one versus all technique" (i.e., the ROC for one class was generated to

806 classify this class against everything else).

808 The classification process and the calculation of the AUC were repeated 100-times to ensure a 809 reliable estimate of the average classification performance. To evaluate the significance of the 810 classifier performance, we used permutation testing. In this test, the response traces were kept

811 the same, but their labels were randomly permutated. After repeating the permutation procedure

812 100-times, we calculated the AUCs for a classifier trained on a dataset with randomly assigned

813 labels and tested on true classes. This approach generated a null distribution, which we used

814 for the empirical p-value calculation (i.e., the proportion of permutations for which the AUC is

815 greater than the score obtained using the original data) ${ }^{39}$ (Table 19).

817 Reporting summary

818

819 Further information on research design is available in the Research Reporting Summary linked

820 to this paper.

824 The data that support the findings of this study will be deposited in the Brain Image Library (BIL;

825 https://www.brainimagelibrary.org/index.html), as required for this BRAIN Initiative-funded

826 project. They will also be available from the corresponding authors upon reasonable request. 


\section{Code availability}

The custom Matlab-based code used for acquisition, processing, and analysis of the data will be 832 from the corresponding authors upon reasonable request.

\section{References}

30. Garcia, A. D. R., Doan, N. B., Imura, T., Bush, T. G. \& Sofroniew, M. V. GFAP-expressing progenitors are the principal source of constitutive neurogenesis in adult mouse forebrain. Nat Neurosci 7, 1233-1241 (2004).

31. Madisen, L. et al. A toolbox of Cre-dependent optogenetic transgenic mice for light-

32. Goldey, G. J. et al. Removable cranial windows for long-term imaging in awake mice. Nat Protoc 9, 2515-2538 (2014).

843 33. Asaad, W. F. \& Eskandar, E. N. A flexible software tool for temporally-precise behavioral 844 control in Matlab. J. Neurosci. Methods 174, 245-258 (2008).

845 34. Asaad, W. F., Santhanam, N., McClellan, S. M. \& Freedman, D. J. High-performance execution of psychophysical tasks with complex visual stimuli in MATLAB. J Neurophys

848 35. Pnevmatikakis, E. A. \& Giovannucci, A. NoRMCorre: An online algorithm for piecewise rigid motion correction of calcium imaging data. J. Neurosci. Methods 291, 83-94 (2017).

850 36. Brenner, M. \& Messing, A. Regulation of GFAP Expression. ASN Neuro 13, 1759091420981206 (2021).

852 37. McQuin, C. et al. CellProfiler 3.0: Next-generation image processing for biology. PLoS Biol. 16, e2005970 (2018).

854 38. Pinheiro, J. \& Bates, D. Mixed-effects models in S and S-PLUS. (2006). 
39. Ojala, M. \& Garriga, G. C. Permutation tests for studying classifier performance. Journal of Machine Learning 11, 1833-1863 (2010).

\section{Acknowledgments}

We thank J.H. Reynolds, T.J. Sejnowski, the U19 A-Team, and members of the Nimmerjahn lab for comments on the manuscript, the Salk machine shop for technical support, and J. Chambers for mouse colony management. This work was primarily supported by the National Institutes of Health (NIH) grant U19NS123719 (A.N.). It was partially supported by the NIH grants U01NS103522 (A.N.), R01NS108034 (A.N) and UL1TR001442 (Altman Clinical \& Translational Research Institute; ACTRI). K.M. was a DFG research fellow and Catharina Foundation postdoctoral scholar. The content is solely the authors' responsibility and does not necessarily

867 represent the official views of the $\mathrm{NIH}$.

\section{Author contributions}

870

K.M. and A.N. conceived and designed the study. K.M. designed and performed the behavioral and imaging experiments with help from R.W.F. and D.D. K.M. designed and performed the statistical analysis with help from ACTRI services and prepared the figures. K.M. and A.N. wrote

874 the text. All authors discussed the results, provided input or edits on the manuscript.

\section{Competing interests}

878 The authors declare no competing interests. 
bioRxiv preprint doi: https://doi.org/10.1101/2021.10.09.463784; this version posted October 10,2021 . The copyright holder for this preprint (which was not certified by peer review) is the author/funder, who has granted bioRxiv a license to display the preprint in perpetuity. It is made available under aCC-BY-ND 4.0 International license.

Extended Data Figures

884

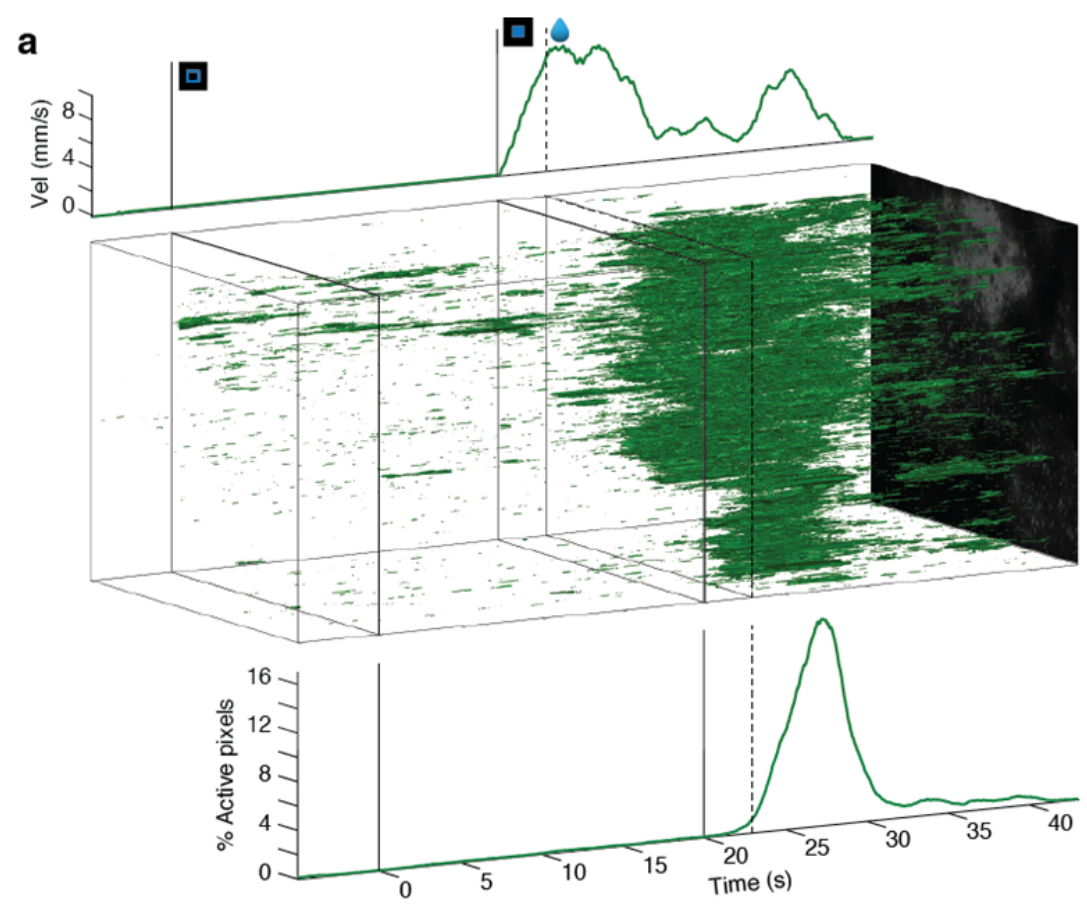

b

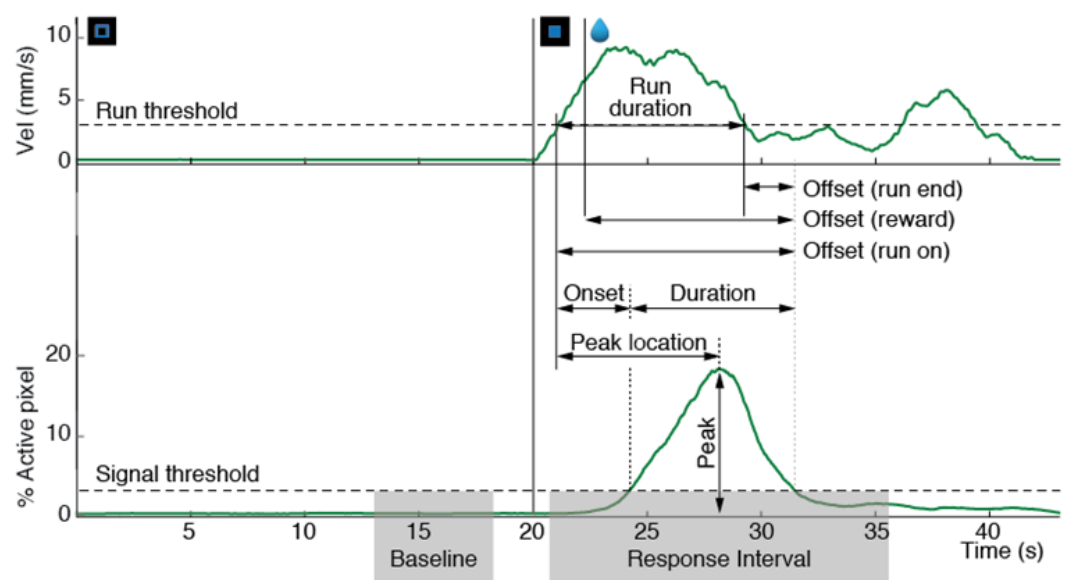


887 Extended Data Fig. 1 | Approach for extracting and analyzing astrocyte syncytium

888 calcium signals.

889 a, The Regions of Activity (ROA) algorithm ${ }^{7}$ was used to extract and characterize astrocyte

890 syncytium calcium signals. The example data shows one representative hit trial. Top, run

891 velocity profile. Center, $x-y-t$ rendering of active pixels detected within the $(\sim 510 \times 640 \mu \mathrm{m}$ field-

892 of-view (FOV). Bottom, the percentage of active pixels over time normalized to all labeled pixels

893 within the FOV. b, Schematic of the astrocyte signal characteristics used for data analysis. Top,

894 run velocity profile. Bottom, astrocyte syncytium calcium signal. 
a

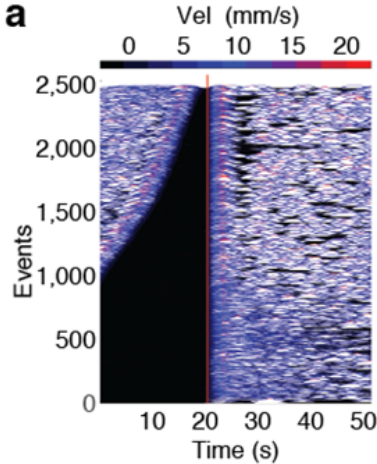

d

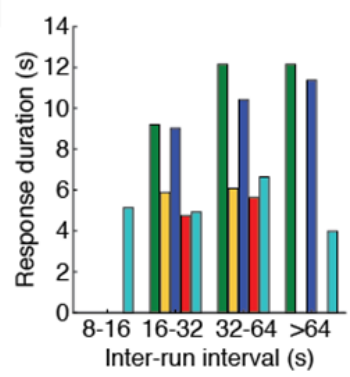

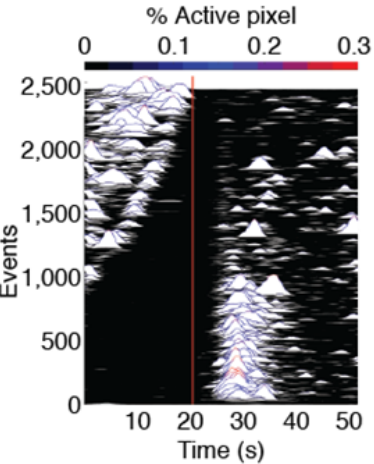

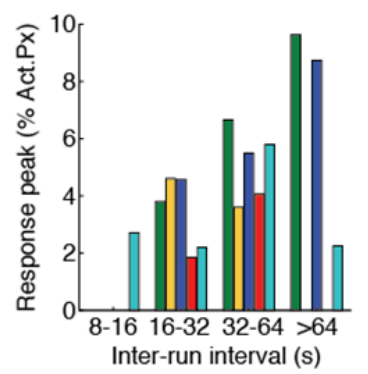

-

b

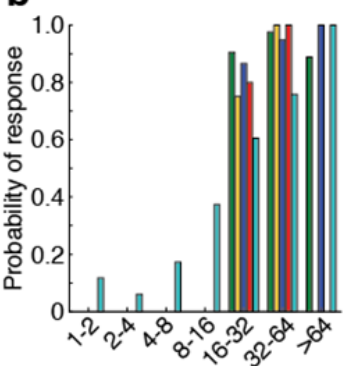

Inter-run interval (s)

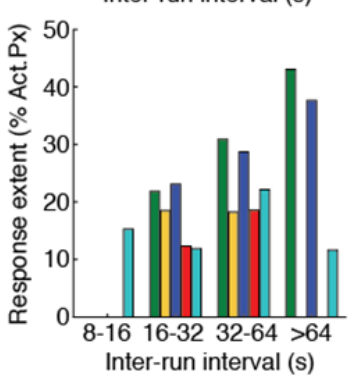

C
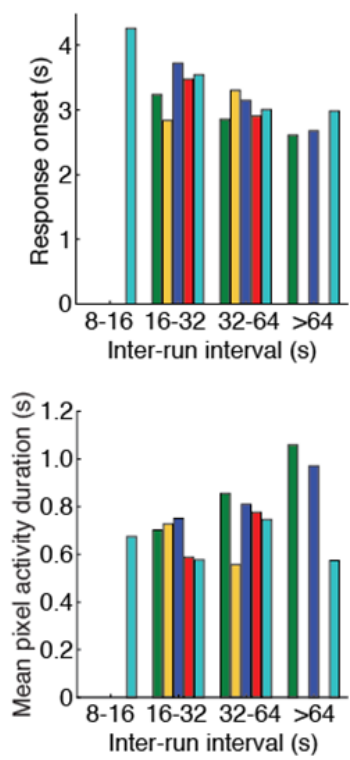

Extended Data Fig. 2 | Astrocyte syncytium response properties depend on the rest period between runs.

a-d, Astrocytes' syncytium calcium response probability, onset, and strength in area M1/M2 depends on the rest period between runs. This inter-run interval dependency was accounted for during data analysis, including only trials with $>15$ s rest periods between runs. a, Paired traces

903 of running activity (left) and syncytium calcium signals (right), ordered by the inter-trial interval

904 and aligned on the current run's onset (red lines). b, Astrocyte syncytium response probability

905 as a function of the inter-run interval and trial type. c, Astrocyte syncytium response onset as a

906 function of the inter-run interval and trial type. d, Astrocytes syncytium response strength, as

907 quantified by response duration, peak, total activation extent, and mean pixel activation duration

908 (from left to right), as a function of the inter-run interval and trial type. 
a

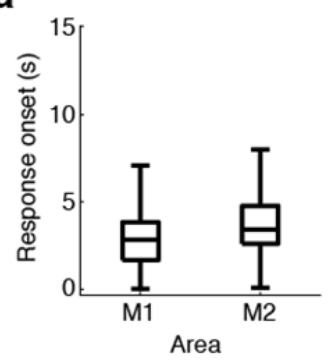

b

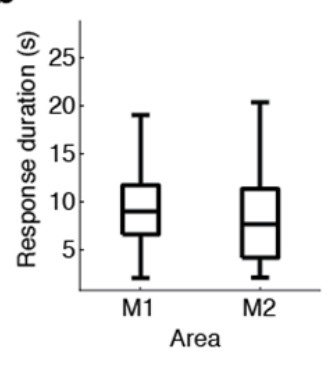

C

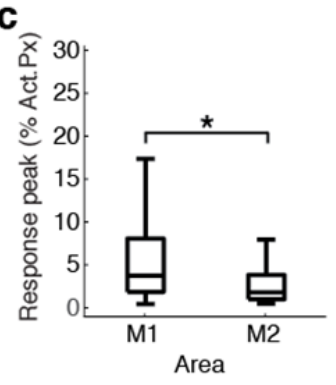

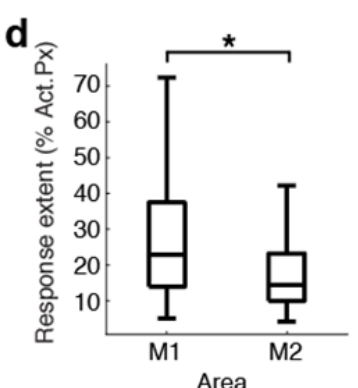

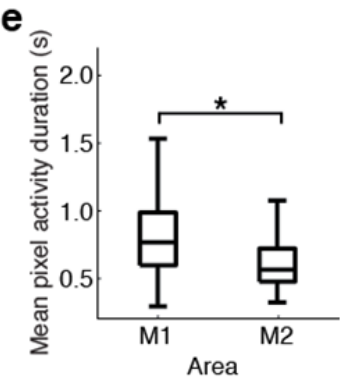

911

912 Extended Data Fig. 3 | Astrocyte syncytium responses show regional differences.

913 a-e, Astrocyte syncytium response onset and duration were comparable between areas M1 and

914 M2 for the different trial types. In contrast, response peak, total activation extent, and mean

915 pixel activation duration were significantly larger in area M1. a, Response onsets. b, Response

916 durations. c, Response peaks. d, Total response extent. e, Mean pixel activation durations.

917

918

919 

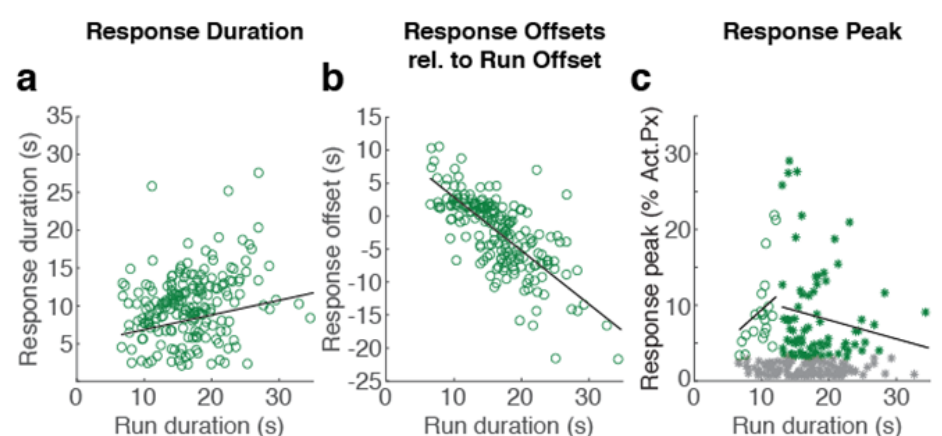

\section{d}
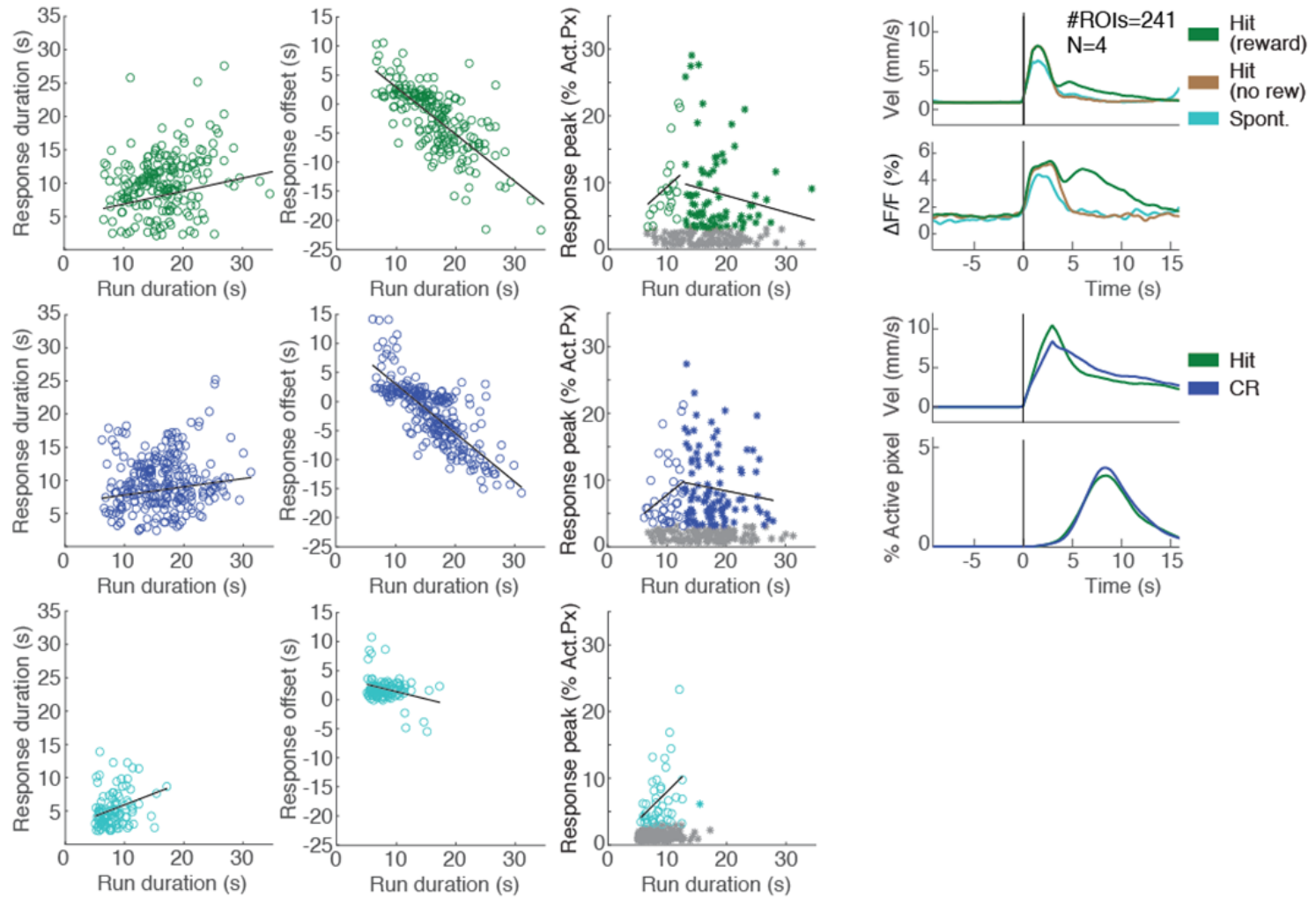

923 Extended Data Fig. 4 | Astrocyte syncytium responses correlate with dopamine signaling

924 in rewarded trials.

925 a, Astrocyte syncytium responses in hit trials, CR trials, and spontaneous runs (top to bottom)

926 increase slightly with run duration. b. Astrocyte syncytium response offsets relative to run offset

927 decrease strongly for longer run durations in hit (top) and CR (center) trials. Response offsets

928 coincide with run offsets in 13-15 s-long runs. In contrast, response offsets for spontaneous

929 runs (bottom) are only slightly modulated by run duration, coinciding mostly with run offsets.

930 c, Astrocyte syncytium response peak varies with run duration for hit trials, CR trials, and

931 spontaneous runs (top to bottom). 13 s-long runs produced the highest response peaks. Linear

932 fits to the data from $<13 \mathrm{~s}$ and $>13 \mathrm{~s}$-long runs showed that response peak values steadily rose 
933 toward the "preferred" $13 \mathrm{~s}$ run duration and declined after that. Trials with response peaks of

$934 \leq 3 \%$ active pixels (gray) were excluded from the linear fits. LME analysis was used to derive fit

935 significance. d, Astrocyte response duration and offset in rewarded hit and CR trials correlated

936 with the period dopamine is present in the extracellular space after reward delivery. Dopamine

937 signals were measured with the genetically encoded indicator dLight1.2 in layer 2/3 of cortical

938 areas M1/M2 during detection task performance ${ }^{17}$. Top, run velocity profiles and corresponding

939 average dLight1.2 transients for rewarded hit trials (green), unrewarded hit trials (brown), and

940 spontaneous runs (cyan) aligned at the run onset. The traces are an average across ROls

941 active during reward (241 ROIs from four mice). Bottom, run velocity profiles and corresponding

942 astrocyte syncytium responses for rewarded hit (198 trials, green) and CR trials (260 trials, blue)

943 aligned at the run onset. 
bioRxiv preprint doi: https://doi.org/10.1101/2021.10.09.463784; this version posted October 10,2021 . The copyright holder for this preprint (which was not certified by peer review) is the author/funder, who has granted bioRxiv a license to display the preprint in perpetuity. It is made available under aCC-BY-ND 4.0 International license.

a kNN Classification of Trial Types; Accuracy $=38 \%$

\begin{tabular}{|c|c|c|c|c|c|c|}
\hline \multirow{7}{*}{ 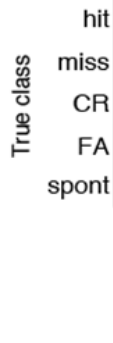 } & 99 & 13 & 56 & 11 & 19 & $50.0 \% 50.0 \%$ \\
\hline & & 5 & 1 & 4 & 7 & $29.4 \% 70.6 \%$ \\
\hline & 115 & 23 & 99 & 15 & 8 & $38.1 \% 61.9 \%$ \\
\hline & 1 & 2 & 1 & 10 & 1 & $66.7 \% 33.3 \%$ \\
\hline & 6 & 38 & 14 & 35 & 13 & $12.3 \% 87.7 \%$ \\
\hline & \begin{tabular}{|l|}
$44.8 \%$ \\
$55.2 \%$
\end{tabular} & $\begin{array}{l}6.2 \% \\
93.8 \%\end{array}$ & & & $\begin{array}{l}27.1 \% \\
72.9 \%\end{array}$ & \\
\hline & hit & $\begin{array}{c}\text { miss } \\
\text { Pre }\end{array}$ & $\begin{array}{r}\mathrm{CR} \\
\text { ilcted }\end{array}$ & & spont & \\
\hline
\end{tabular}

b kNN Classification of Performance Level; Accuracy $=62 \%$
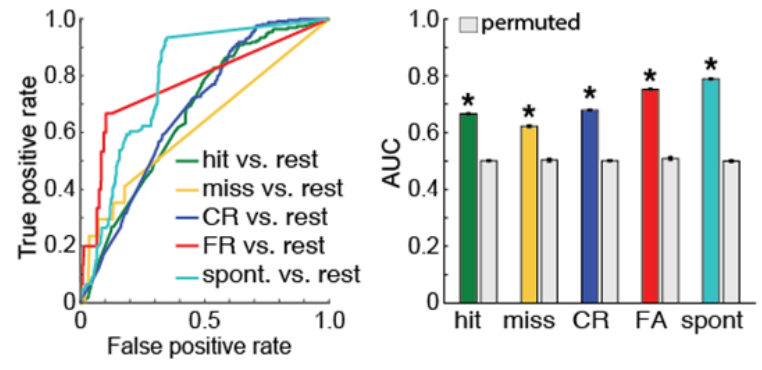
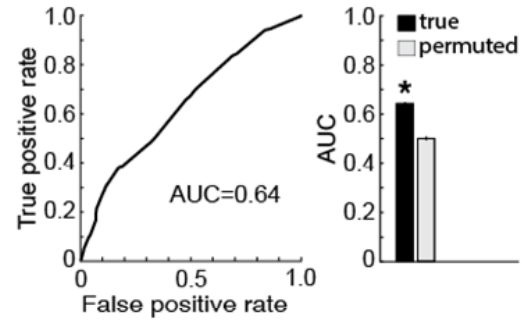

C

kNN Classification of Run Duration, Spont. Runs; Accuracy=90\%

\begin{tabular}{|c|c|c|c|c|c|}
\hline \multirow{3}{*}{ 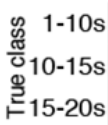 } & 70 & 11 & 3 & $83.3 \%$ & $16.7 \%$ \\
\hline & 2 & 16 & 1 & $84.2 \%$ & $15.8 \%$ \\
\hline & & & 3 & $100 \%$ & \\
\hline & \multicolumn{3}{|c|}{$97.2 \% 59.3 \% 42.9 \%$} & & \\
\hline & $2.8 \%$ & $40.7^{\circ}$ & $57.1 \%$ & & \\
\hline & $\begin{array}{r}\text { 1-10s } \\
\text { Pre }\end{array}$ & $\begin{array}{l}10-15 \\
\text { licted }\end{array}$ & $\begin{array}{l}15-20 \mathrm{~s} \\
\text { ass }\end{array}$ & & \\
\hline
\end{tabular}
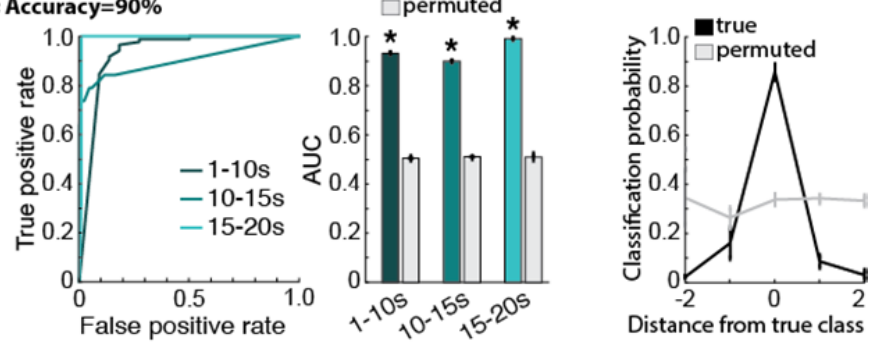

d

\begin{tabular}{|c|c|c|c|c|}
\hline \multirow{3}{*}{ 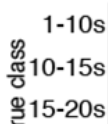 } & 44 & 15 & 13 & 12 \\
\hline & 23 & 55 & 30 & 24 \\
\hline & 14 & 45 & 57 & 57 \\
\hline${ }^{\prime} 20-30 \mathrm{~s}$ & 5 & 17 & 19 & 56 \\
\hline & \begin{tabular}{|l}
$58.2 \%$ \\
$48.8 \%$
\end{tabular} & $\begin{array}{l}41.7 \% \\
58.3 \%\end{array}$ & \begin{tabular}{|l|}
$47.9 \%$ \\
$52.1 \%$
\end{tabular} & $\begin{array}{l}37.6 \% \\
62.4 \%\end{array}$ \\
\hline & $1-10 \mathrm{~s}$ & $\begin{array}{l}10-15 \mathrm{~s} \\
\text { Predicte }\end{array}$ & $\begin{array}{l}15-20 \mathrm{~s} \\
\text { ed class }\end{array}$ & $20-30 \mathrm{~s}$ \\
\hline
\end{tabular}

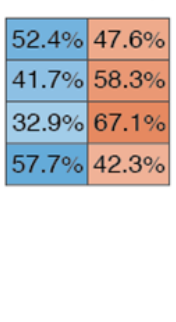

$52.4 \% 47.6 \%$

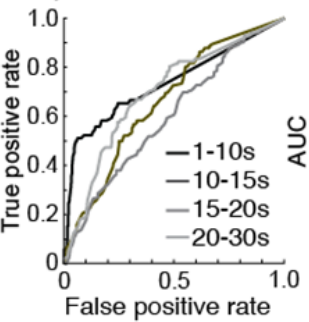

Distance from true class 
947 Extended Data Fig. 5 | Behavioral aspects can be decoded from astrocytic syncytium

948 responses using machine learning approaches.

949 a-d, Astrocyte syncytium signals carry behavioral information as indicated by the k-nearest

950 neighbor (kNN) classifier's prediction accuracy. For each classification, confusion matrices,

951 receiver-operating characteristic (ROC) curves and area under the ROC curves (AUC) for the

952 classifier's output, and statistical analysis of significance based on permutation tests are shown

953 (left to right). Error bars indicate s.e.m. a, The kNN classifier decoded the trial type from

954 astrocyte syncytium responses significantly above chance level. Most confusions happened

955 between hit and CR trials. The decoding performance was worst for miss trials. b, Animal

956 performance level could be significantly decoded from astrocyte syncytium signals.

957 c, Spontaneous run durations could be decoded from astrocyte syncytium responses. d, Task-

958 related run duration could be decoded from astrocyte syncytium responses. Confusions were

959 more likely between neighboring run duration classes. Far-right plots in $c$ and $d$ show the

960 decoding probabilities for a given run duration class as a function of distance from the true class

961 (black line). The gray line depicts the average decoding probabilities based on permutation tests

962 (see Methods). 
bioRxiv preprint doi: https://doi.org/10.1101/2021.10.09.463784; this version posted October 10,2021 . The copyright holder for this preprint (which was not certified by peer review) is the author/funder, who has granted bioRxiv a license to display the preprint in perpetuity. It is made available under aCC-BY-ND 4.0 International license.
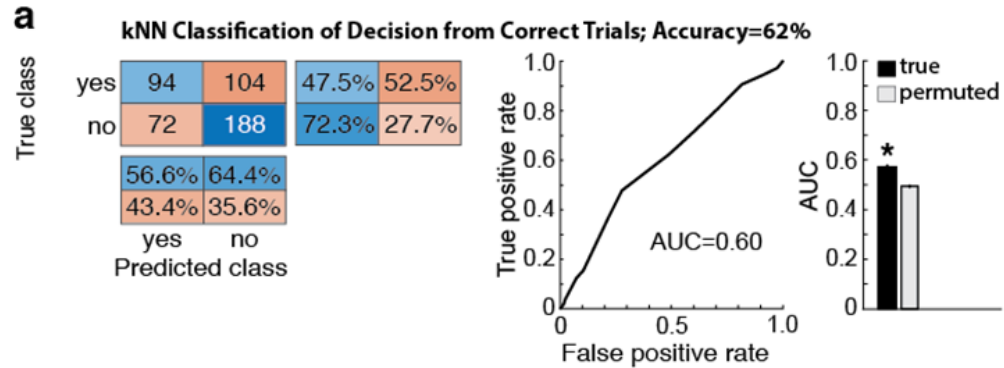

b
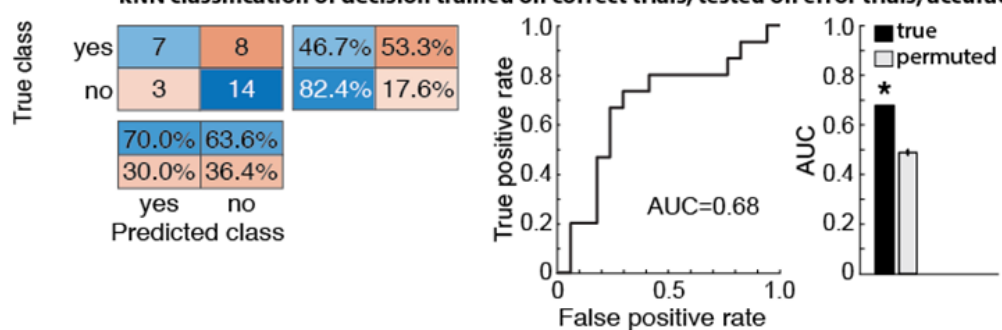

C kNN classification of stimulus intensity from hit trials; accuracy $=65 \%$

\begin{tabular}{|c|c|c|c|c|}
\hline \multirow{4}{*}{ 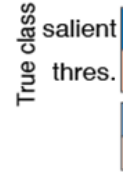 } & 97 & 33 & $74.6 \%$ & $25.4 \%$ \\
\hline & 36 & 32 & $47.1 \%$ & $52.9 \%$ \\
\hline & $72.9 \%$ & $49.2 \%$ & & \\
\hline & $27.1 \%$ & $50.8 \%$ & & \\
\hline
\end{tabular}
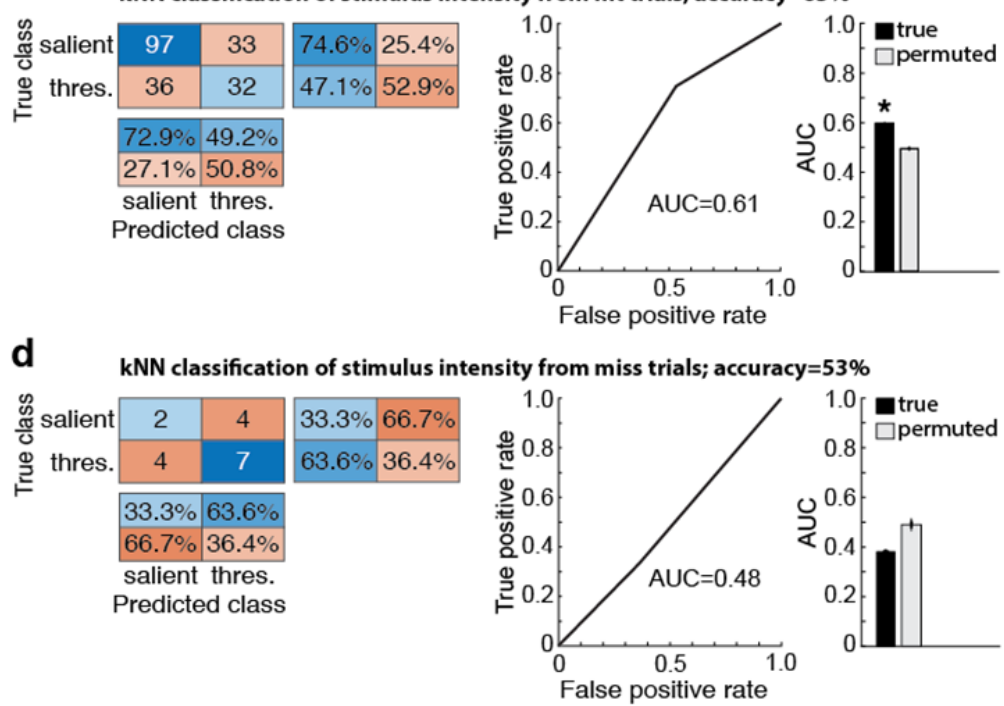
967 Extended Data Fig. 6 | Astrocyte syncytium responses are behaviorally relevant.

968 a-d, Separate classifications of correct and error trials reveal that information encoded by

969 astrocyte syncytium calcium responses is relevant for the animal's behavior. For each

970 classification, confusion matrices, receiver-operating characteristic (ROC) curves for the

971 classifier's output and Area under the ROC curves (AUC), and statistical analysis of significance

972 based on permutation tests are shown (left to right). Error bars indicate s.e.m. a, Decoding the

973 animal's decision about stimulus presence or absence was possible from astrocytes' syncytium

974 responses to hit and CR trials. b, Decoding the animal's decision was also possible when the

975 classifier was trained on correct (hit and CR) but tested on erroneous (miss and FA) trials.

976 Significant prediction of the animal's decision (miss-'no', FA-'yes') was confirmed by the AUC

977 value. This value was significantly higher than AUC values obtained on a training set with

978 randomly shuffled class labels. c, Information about stimulus intensity could be significantly

979 decoded from astrocyte syncytium responses to hit trials. d, Miss trials lack behaviorally

980 relevant sensory information, as the decoder fails to classify error trials according to stimulus

981 intensity.

982

983

984 

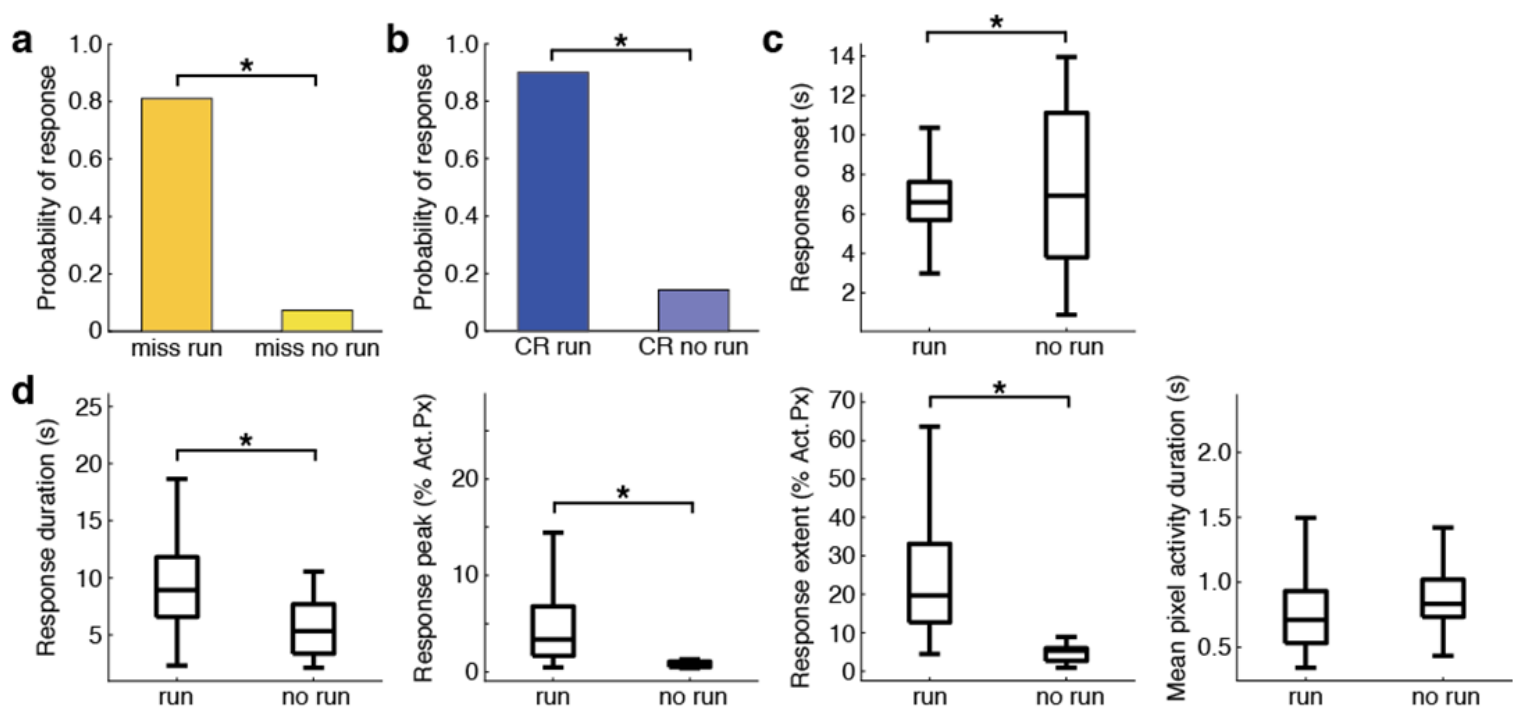

987 Extended Data Fig. 7 | Astrocyte syncytium responses depend on run occurrence.

988 a-d, Astrocyte syncytium responses are significantly different in miss and CR trials with or

989 without a subsequent run. During miss and CR trials, the animals remain still throughout the

990 stimulus presentation phase. However, they start running in most CR trials during reward

991 consumption and occasionally miss trials after stimulus offset. These trial types allowed for the

992 comparison of trials with and without a run. a, Astrocyte syncytium response probability for miss

993 trials with and without a subsequent run. b, Astrocyte syncytium response probability for CR

994 trials with and without a subsequent run. c, Response onsets for 'run' and 'no run' trials

995 averaged across miss and CR trial types aligned at stimulus onset. d, Astrocyte syncytium

996 response strength, as quantified by response duration, peak, total activation extent, and mean

997 pixel activation (from left to right), for 'run' and 'no run' trials averaged across miss and CR trial

998 types.

999

1000 


\section{Extended Data Tables}

Table 1 | Numbers of trials included in the syncytium response analysis.

1004 1005 1006 1007

\begin{tabular}{ccccccc} 
Hit & \multicolumn{2}{c}{ Miss } & \multicolumn{2}{c}{ CR } & FA & Spont. \\
\hline & run & no run & run & no run & & \\
\hline 98 & 17 & 253 & 260 & 6 & 15 & 106 \\
\hline 23 & 4 & 20 & 30 & 1 & 3 & 61
\end{tabular}

\section{Table 2 | Generalized linear mixed-effects model for astrocyte syncytium response probability. All} qualifying trials (Table 1), followed by a run, were included in the analysis. Degrees of freedom: 717.

\begin{tabular}{lccc} 
Behavioral Variable & Coefficient & Standard Error & p-value \\
\hline Trial type hit vs. miss & 3.48 & 2.93 & 0.23 \\
\hline Trial type hit vs. CR & -0.51 & 1.03 & 0.62 \\
\hline Trial type hit vs. FA & -2.94 & 3.61 & 0.42 \\
\hline Trial type hit vs. spont. & -2.96 & 1.12 & 0.01 \\
\hline Trial type miss vs. CR & 0.66 & 0.64 & 0.30 \\
\hline Trial type miss vs. FA & -0.01 & 0.88 & 0.99 \\
\hline Trial type miss vs. spont. & -0.83 & 0.61 & 0.18 \\
\hline Trial type CR vs. FA & -0.68 & 0.70 & 0.33 \\
\hline Trial type CR vs. spont. & -1.49 & 0.29 & $5 \cdot 10^{-7}$ \\
\hline Trial type FA vs. spont. & -0.81 & 0.68 & 0.23 \\
\hline Run duration & 0.12 & 0.05 & 0.02 \\
\hline Inter-run interval & 0.04 & 0.01 & $5 \cdot 10^{-4}$ \\
\hline Trial type miss: Run duration & -0.44 & 0.34 & 0.19 \\
\hline Trial type CR: Run duration & 0.02 & 0.07 & 0.74 \\
\hline Trial type FA: Run duration & 0.43 & 0.51 & 0.40 \\
\hline Trial type spont.: Run duration & 0.31 & 0.12 & 0.01
\end{tabular}


Table 3 | Linear mixed-effects model for astrocyte syncytium response onset. All qualifying trials (Table 1) with a run and significant syncytium response were included. Degrees of freedom: 590.

\section{Coefficient Standard Error p-value}

\begin{tabular}{lllc}
\hline Trial type hit vs. miss & -0.07 & 0.40 & 0.85 \\
\hline Trial type hit vs. CR & 0.81 & 0.15 & $1 \cdot 10^{-7}$ \\
\hline Trial type hit vs. FA & 0.64 & 0.41 & 0.12 \\
\hline Trial type hit vs. spont. & 0.60 & 0.20 & 0.002 \\
\hline Trial type miss vs. CR & 0.88 & 0.40 & 0.03 \\
\hline Trial type miss vs. FA & 0.71 & 0.54 & 0.19 \\
\hline Trial type miss vs. spont. & 0.68 & 0.40 & 0.09 \\
\hline Trial type CR vs. FA & -0.17 & 0.41 & 0.67 \\
\hline Trial type CR vs. spont. & -0.21 & 0.19 & 0.29 \\
\hline Trial type FA vs. spont. & 0.03 & 0.42 & 0.94 \\
\hline Inter-run interval & 0.04 & 0.01 & $5 \cdot 10^{-4}$
\end{tabular}

Table 4 | Linear mixed-effects model for astrocyte syncytium response duration. All qualifying trials (Table 1) with a run and significant syncytium response were included. Degrees of freedom: 589.

Behavioral Variable

Coefficient Standard Error p-value

\begin{tabular}{lccc}
\hline Trial type hit vs. miss & -2.04 & 0.91 & 0.02 \\
\hline Trial type hit vs. CR & -0.63 & 0.33 & 0.06 \\
\hline Trial type hit vs. FA & -3.82 & 0.94 & $5 \cdot 10^{-5}$ \\
\hline Trial type hit vs. spont. & -3.16 & 0.48 & $1 \cdot 10^{-10}$ \\
\hline Trial type miss vs. CR & 1.40 & 0.90 & 0.12 \\
\hline Trial type miss vs. FA & -1.78 & 1.19 & 0.13 \\
\hline Trial type miss vs. spont. & -1.12 & 0.88 & 0.20 \\
\hline Trial type CR vs. FA & -3.19 & 0.93 & $6 \cdot 10^{-4}$ \\
\hline Trial type CR vs. spont. & -2.52 & 0.47 & $9 \cdot 10^{-8}$ \\
\hline Trial type FA vs. spont. & -0.66 & 0.92 & 0.47 \\
\hline Run duration & 0.17 & 0.03 & $3 \cdot 10^{-7}$ \\
\hline Inter-run interval & 0.03 & 0.01 & $1 \cdot 10^{-6}$
\end{tabular}


Table 5 | Linear mixed-effects model for astrocyte syncytium response peak. All qualifying trials (Table 1) with a run and significant syncytium response were included. Degrees of

1025 freedom: 588.

\begin{tabular}{lccc} 
Behavioral Variable & Coefficient & Standard Error & p-value \\
\hline Trial type hit vs. miss & 0.04 & 0.22 & 0.87 \\
\hline Trial type hit vs. CR & -0.08 & 0.08 & 0.31 \\
\hline Trial type hit vs. FA & -0.61 & 0.22 & $6 \cdot 10^{-3}$ \\
\hline Trial type hit vs. spont. & -0.33 & 0.10 & $2 \cdot 10^{-3}$ \\
\hline Trial type miss vs. CR & -0.12 & 0.21 & 0.58 \\
\hline Trial type miss vs. FA & -0.65 & 0.3 & 0.03 \\
\hline Trial type miss vs. spont. & -0.36 & 0.22 & 0.09 \\
\hline Trial type CR vs. FA & -0.53 & 0.22 & 0.02 \\
\hline Trial type CR vs. spont. & -0.24 & 0.10 & 0.02 \\
\hline Trial type FA vs. spont. & 0.28 & 0.22 & 0.20 \\
\hline Area (M2) & -0.48 & 0.19 & $1 \cdot 10^{-83}$ \\
\hline Performance (high) & -0.29 & 0.08 & $1 \cdot 10^{-4}$ \\
\hline Inter-run interval & 0.01 & 0.001 & $7 \cdot 10^{-13}$
\end{tabular}

Table 6 | Linear mixed-effects model for astrocyte syncytium response extent. All qualifying trials

(Table 1) with a run and significant syncytium response were included. Degrees of freedom: 588.

Behavioral Variable Coefficient Standard Error p-value

\begin{tabular}{lccc}
\hline Trial type hit vs. miss & -0.31 & 0.14 & 0.03 \\
\hline Trial type hit vs. CR & -0.12 & 0.05 & 0.03 \\
\hline Trial type hit vs. FA & -0.64 & 0.15 & $2 \cdot 10^{-5}$ \\
\hline Trial type hit vs. spont. & -0.54 & 0.07 & $3 \cdot 10^{-14}$ \\
\hline Trial type miss vs. CR & 0.19 & 0.14 & 0.18 \\
\hline Trial type miss vs. FA & -0.33 & 0.19 & 0.08 \\
\hline Trial type miss vs. spont. & -0.23 & 0.14 & 0.10 \\
\hline Trial type CR vs. FA & -0.52 & 0.14 & $4 \cdot 10^{-4}$ \\
\hline Trial type CR vs. spont. & -0.42 & 0.08 & $1 \cdot 10^{-9}$ \\
\hline Trial type FA vs. spont. & 0.10 & 0.15 & 0.50 \\
\hline Area (M2) & -0.26 & 0.12 & 0.03 \\
\hline Performance (high) & -0.18 & 0.05 & $2 \cdot 10^{-4}$ \\
\hline Inter-run interval & 0.008 & 0.001 & $6 \cdot 10^{-14}$
\end{tabular}


Table 7 | Linear mixed-effects model for mean pixel activity duration. All qualifying trials (Table 1) with a run and significant syncytium response were included. Degrees of freedom: 588.

\begin{tabular}{lccc} 
Behavioral Variable & Coefficient & Standard Error & p-value \\
\hline Trial type hit vs. miss & -0.09 & 0.07 & 0.23 \\
\hline Trial type hit vs. CR & -0.05 & 0.03 & 0.60 \\
\hline Trial type hit vs. FA & -0.25 & 0.07 & $7 \cdot 10^{-4}$ \\
\hline Trial type hit vs. spont. & -0.17 & 0.03 & $5 \cdot 10^{-7}$ \\
\hline Trial type miss vs. CR & 0.03 & 0.07 & 0.62 \\
\hline Trial type miss vs. FA & -0.16 & 0.09 & 0.09 \\
\hline Trial type miss vs. spont. & -0.09 & 0.07 & 0.21 \\
\hline Trial type CR vs. FA & -0.19 & 0.07 & 0.006 \\
\hline Trial type CR vs. spont. & -0.12 & 0.03 & $2 \cdot 10^{-4}$ \\
\hline Trial type FA vs. spont. & 0.07 & 0.07 & 0.31 \\
\hline Area (M2) & -0.20 & 0.09 & 0.02 \\
\hline Performance (high) & -0.07 & 0.02 & 0.005 \\
\hline Inter-run interval & 0.003 & $5 \cdot 10^{-4}$ & $3 \cdot 10^{-10}$
\end{tabular}

Table 8 | Linear mixed-effects models for astrocyte syncytium response strength probing qualifying task trials (Table 1) with a run and significant syncytium response were included. Degrees of freedom: 486.

\begin{tabular}{llll} 
Behavioral Variable & Coefficient & Standard Error & p-value \\
\hline Response duration & & & \\
\hline Correct/Error trials & -2.66 & 0.73 & $3 \cdot 10^{-4}$ \\
\hline Run duration & 0.16 & 0.04 & $5 \cdot 10^{-6}$ \\
\hline Inter-run interval & 0.03 & 0.007 & $6 \cdot 10^{-6}$ \\
\hline Extent & & \\
\hline Correct/Error trials & -0.40 & 0.10 & $2 \cdot 10^{-4}$ \\
\hline Performance (high) & -0.15 & 0.05 & 0.005 \\
\hline Inter-run interval & 0.01 & 0.001 & $2 \cdot 10^{-12}$ \\
\hline Mean pixel activity duration & & & \\
\hline Correct/Error trials & -0.11 & 0.05 & 0.03 \\
\hline Area (M2) & -0.21 & 0.09 & 0.03 \\
\hline Inter-run interval & 0.003 & $5 \cdot 10^{-4}$ & $2 \cdot 10^{-9}$
\end{tabular}


Table 10 | Linear mixed-effects model testing the relationship between astrocyte syncytium response and stimulus intensity. Only hit trials with a run and a significant astrocyte syncytium response were included in the analysis (Table 1). Degrees of freedom: 240.

\begin{tabular}{lccc} 
Behavioral Variable & Coefficient & Standard Error & p-value \\
\hline Response onset & & & \\
\hline Stimulus type (thresh.) & 0.36 & 0.19 & 0.05 \\
\hline Inter-run interval & -0.01 & 0.01 & 0.05
\end{tabular}

Table 11 | Linear mixed-effects models examining the astrocyte syncytium response's probability, onset, and strength with respect to run occurrence. All trials with a significant syncytium response were included (Table 1). All trials were aligned at trial onset.

\begin{tabular}{|c|c|c|c|c|}
\hline Behavioral Variable & Coefficient & Standard Error & p-value & $\begin{array}{c}\text { Degrees of } \\
\text { Freedom } \\
\end{array}$ \\
\hline \multicolumn{5}{|l|}{ Probability (miss trials) } \\
\hline Run/No run (run) & 4.2 & 0.74 & $1 \cdot 10^{-12}$ & 292 \\
\hline \multicolumn{5}{|l|}{ Probability (CR trials) } \\
\hline Run/No run (run) & 4.93 & 1.32 & $2 \cdot 10^{-4}$ & 295 \\
\hline \multicolumn{5}{|l|}{ Onset } \\
\hline Run/No run (run) & -2.10 & 0.43 & $2 \cdot 10^{-5}$ & 297 \\
\hline \multicolumn{5}{|l|}{ Response duration } \\
\hline Run/No run (run) & 3.50 & 0.86 & $6 \cdot 10^{-5}$ & 294 \\
\hline \multicolumn{5}{|l|}{ Response peak } \\
\hline Run/No run (run) & 1.45 & 0.22 & $9 \cdot 10^{-11}$ & 294 \\
\hline \multicolumn{5}{|l|}{ Response extent } \\
\hline Run/No run (run) & 1.75 & 0.14 & $2 \cdot 10^{-29}$ & 297 \\
\hline \multicolumn{5}{|c|}{ Mean pixel activity duration } \\
\hline Run/No run (run) & -0.09 & 0.07 & 0.25 & 297 \\
\hline
\end{tabular}
encoding ('yes' - hit and FA trials; 'no' - CR and miss trials). The categorical variable decision was included instead of the trial type. All qualifying task trials (Table 1) with a run and significant syncytium response were included. Degrees of freedom: 487.

Behavioral Variable

Coefficient

Standard Error p-value

Response onset

\begin{tabular}{lccc}
\hline Decision (no) & 0.68 & 0.15 & $5 \cdot 10^{-6}$ \\
\hline Inter-run interval & -0.01 & 0.003 & $4 \cdot 10^{-4}$
\end{tabular}

$$
-0.01
$$


Table 12 | Linear mixed-effects model for astrocyte syncytium response duration, testing for the effect of run duration in hit trials, CR trials, and spontaneous runs. All qualifying trials (Table 1) with a run and significant syncytium response for the respective trial types were included.

\begin{tabular}{lcccc} 
Behavioral Variable & Coefficient & Standard Error & p-value & $\begin{array}{c}\text { Degrees of } \\
\text { Freedom }\end{array}$ \\
\hline Hit trials & & & & \\
\hline Run duration & 0.19 & 0.05 & $4 \cdot 10^{-4}$ & 195 \\
\hline Inter-run interval & 0.06 & 0.01 & $9 \cdot 10^{-5}$ & 195 \\
\hline CR trials & & & & \\
\hline Run duration & 0.12 & 0.05 & 0.01 & 257 \\
\hline Inter-run interval & 0.02 & 0.01 & 0.003 & 257 \\
\hline Spontaneous runs & & & & \\
\hline Run duration & 0.34 & 0.10 & $6 \cdot 10^{-4}$ & 104
\end{tabular}

Table 13 | Linear mixed-effects model for astrocyte syncytium response onset, testing for the effect of run duration in hit trials, CR trials, and spontaneous runs. All qualifying trials (Table 1) with a run and significant syncytium response for the respective trial types were included.

\begin{tabular}{|c|c|c|c|c|}
\hline Behavioral Variable & Coefficient & Standard Error & p-value & $\begin{array}{c}\text { Degrees o } \\
\text { Freedom }\end{array}$ \\
\hline
\end{tabular}

Hit trials

\begin{tabular}{lcccc}
\hline Run duration & -0.009 & 0.02 & 0.61 & 195 \\
\hline Inter-run interval & -0.01 & 0.005 & 0.04 & 195 \\
\hline CR trials & & & & \\
\hline Run duration & 0.04 & 0.02 & 0.11 & 257 \\
\hline Inter-run interval & -0.01 & 0.004 & 0.006 & 257 \\
\hline Spontaneous runs & & & & \\
\hline Run duration & 0.42 & 0.06 & $3 \cdot 10^{-11}$ & 103 \\
\hline Inter-run interval & -0.02 & 0.009 & 0.40 & 103
\end{tabular}


Table 14 | Linear mixed-effects model for the astrocyte syncytium response's peak location testing for the effect of run duration in hit trials, CR trials, and spontaneous runs. All qualifying trials (Table 1) with a run and significant syncytium response for the respective trial type were included.

\begin{tabular}{lcccc} 
Behavioral Variable & Coefficient & Standard Error & $\begin{array}{c}\text { p-value } \\
\text { Hit trials }\end{array}$ & $\begin{array}{c}\text { Degrees of } \\
\text { Freedom }\end{array}$ \\
\hline Run duration & 0.04 & 0.03 & 0.21 & 196 \\
\hline CR trials & & & & \\
\hline Run duration & 0.11 & 0.03 & $4 \cdot 10^{-5}$ & 258 \\
\hline Spontaneous runs & & & & \\
\hline Run duration & 0.66 & 0.06 & $2 \cdot 10^{-18}$ & 104
\end{tabular}

Table 15 | Linear mixed-effects model for astrocyte syncytium response offset aligned on run onset testing for the effect of run duration in hit trials, CR trials, and spontaneous runs. All qualifying trials (Table 1 ) with a run and significant syncytium response for the respective trial types were included.

Behavioral Variable $\quad$ Coefficient $\quad$ Standard Error $\quad$ p-value $\begin{gathered}\text { Degrees of } \\ \text { Freedom }\end{gathered}$

\section{Hit trials}

\begin{tabular}{lllll}
\hline Run duration & 0.18 & 0.05 & $3 \cdot 10^{-4}$ & 195 \\
\hline Inter-run interval & 0.50 & 0.01 & $4 \cdot 10^{-4}$ & 195 \\
\hline CR trials & & & & \\
\hline Run duration & 0.16 & 0.04 & $6 \cdot 10^{-5}$ & 257 \\
\hline Inter-run interval & 0.01 & 0.006 & 0.05 & 257 \\
\hline Spontaneous runs & & & & \\
\hline Run duration & 0.75 & 0.08 & $8 \cdot 10^{-16}$ & 104
\end{tabular}


Table 18 | Optimized hyperparameters used in the kNN classification analyses.

\begin{tabular}{lccc} 
Classification & Nr. neighbors & Distance & Distance weight \\
\hline Rewarded trials/ spontaneous runs & 7 & correlation & equal \\
\hline Rewarded/ error, non-rewarded & 7 & correlation & equal \\
\hline Trial types (hit, miss, CR, FA, spont.) & 9 & correlation & equal \\
\hline Performance & 11 & jaccard & equal \\
\hline Run duration & 5 & cosine & equal \\
\hline Decision & 50 & correlation & inverse
\end{tabular}

1114 correlation: linear correlation between observations (data were treated as a sequence of values) 1115 jaccard: Jaccard coefficient (the percentage of nonzero coordinates that differ)

Table 16 | Linear mixed-effects model for astrocyte syncytium response offset aligned at the run offset, testing for the effect of run duration in hit trials, CR trials, and spontaneous runs. All qualifying trials (Table 1) with a run and significant syncytium response for the respective trial types were included.

Table 17 | Linear mixed-effects model for determining the relationship between syncytium response peaks and run duration. All qualifying trials (Table 1) with a run and significant syncytium response ( $>3 \%$ active pixels peak value) were included.

Behavioral Variable Coefficient Standard Error

Degrees of Hit trials

\begin{tabular}{ccccc}
\hline Run duration $<13 \mathrm{~s}$ & 0.15 & 0.04 & 0.002 & 19 \\
\hline Run duration $>13 \mathrm{~s}$ & -0.02 & 0.01 & 0.12 & 70 \\
\hline CR trials & & & & \\
\hline Run duration $<13 \mathrm{~s}$ & 0.08 & 0.04 & 0.06 & 37 \\
\hline Run duration $>13 \mathrm{~s}$ & -0.02 & 0.01 & 0.27 & 99 \\
\hline Spontaneous runs & & & & \\
\hline Run duration $<13 \mathrm{~s}$ & 0.1 & 0.04 & 0.01 & 39
\end{tabular}

\begin{tabular}{lcccc} 
Behavioral Variable & Coefficient & Standard Error & p-value & Freedom \\
\hline Hit trials & & & & \\
\hline Run duration & -0.82 & 0.05 & $2 \cdot 10^{-37}$ & 195 \\
\hline Inter-run interval & 0.50 & 0.10 & $5 \cdot 10^{-4}$ & 195 \\
\hline CR trials & & & \\
\hline$\quad$ Run duration & -0.84 & 0.04 & $2 \cdot 10^{-59}$ & 257 \\
\hline$\quad$ Inter-run interval & 0.01 & 0.006 & 0.05 & 257 \\
\hline Spontaneous runs & & & & \\
\hline Run duration & -0.25 & 0.08 & 0.002 & 104
\end{tabular}


cosine: cosine of the angle between observations (data were treated as vectors)

Table 19 | Permutation tests evaluating the kNN classifier's decoding performance. All qualifying trials (Table 1) with a run and significant syncytium response for the respective trial types were included.

Classification mean AUC Standard Error p-value

\begin{tabular}{|c|c|c|c|}
\hline Rewarded and spont. trials & 0.88 & $9 \cdot 10^{-9}$ & 0 \\
\hline Rewarded and erroneous trials & 0.83 & 0.002 & 0 \\
\hline Performance level (all trials used) & 0.64 & 0.001 & 0 \\
\hline \multicolumn{4}{|l|}{ Trial types (all trials used) } \\
\hline hit & 0.67 & 0.002 & 0 \\
\hline miss & 0.63 & 0.006 & 0.02 \\
\hline $\mathrm{CR}$ & 0.68 & 0.001 & 0 \\
\hline $\mathrm{FA}$ & 0.75 & 0.004 & 0.008 \\
\hline spont. & 0.79 & 0.001 & 0 \\
\hline \multicolumn{4}{|l|}{ Run duration, spont. runs } \\
\hline $1-10 \mathrm{~s}$ & 0.93 & 0.001 & 0 \\
\hline $10-15 s$ & 0.90 & 0.002 & 0 \\
\hline $15-20 \mathrm{~s}$ & 0.99 & 0.001 & 0.02 \\
\hline \multicolumn{4}{|l|}{ Run duration, task trials } \\
\hline $1-10 \mathrm{~s}$ & 0.72 & 0.002 & 0 \\
\hline $10-15 s$ & 0.66 & 0.001 & 0 \\
\hline $15-20 \mathrm{~s}$ & 0.58 & 0.002 & 0.01 \\
\hline $20-30 s$ & 0.68 & 0.001 & 0 \\
\hline \multicolumn{4}{|l|}{ Decision } \\
\hline correct trials decoded from correct trials & 0.58 & 0.001 & 0 \\
\hline error trials decoded from correct trials & 0.68 & - & 0.03 \\
\hline \multicolumn{4}{|l|}{ Stimulus } \\
\hline correct trials decoded from correct trials & 0.59 & 0.002 & 0.01 \\
\hline error trials decoded from error trials & 0.38 & 0.007 & 0.51 \\
\hline
\end{tabular}

